\title{
La transformación del juego problemático en España
}

\section{The Transformation of Problematic Gambling/Gaming in Spain}

\author{
José Antonio Gómez Yáñez \\ Universidad Carlos III (Madrid). Departamento de Análisis Social. \\ Estudio de Sociología Consultores \\ jagy@telefonica.net
}

\section{RESUMEN}

El $0,3 \%$ de los residentes en España de 18 a 75 años se puede considerar en niveles de juego problemático. Es una tasa de las más bajas de Europa Occidental, similar a las de Nueva Zelanda 0 Canadá e inferior a Estados Unidos, Australia y los países asiáticos. Desde 1990, se ha reducido y se ha transformado. De incidir en personas de clases medias y bajas de edades intermedias atraídas por las tragamonedas, está pasando a jóvenes que juegan online. Como en todo el mundo, afecta sobre todo a los hombres. La población implicada es tan reducida que no se puede hablar de sectores proclives al juego problemático, sino de individuos en los que surge el problema. En España no hay un problema social de juego problemático, hay problemas individuales que requieren políticas públicas muy precisas y mantener prácticas de juego responsable en las empresas.

Palabras clave: Juego, Juego patológico, Juego problemático, Juego responsable.

\section{ABSTRACT}

The $0.3 \%$ of the Spanish population aged 18 to 75 can be considered to engage in problematic levels of gambling. This is one of the lowest gambling rates in western Europe. Since 1990, Spain's gambling rate has fallen and undergone a drastic transformation. From being a mainly middle- or working-class problem among the middle-aged, gambling has shifted to young people engaging in online gaming. As is the case worldwide, it is a problem that chiefly affects men. Gambling affects such a small proportion of the population that it is difficult to identify sectors of the population prone to problematic gambling; rather, it is a problem that emerges in certain individuals. Spain does not have a social problem in gambling; there are, however, individual problems requiring very specific public policies, as well as a need for businesses to uphold responsible gambling practices.

Keywords: Gaming, gambling, pathologic gambling, problematic gamgling, responsible gaming.

*Autor para correspondencia / Corresponding author: Gómez Yáñez, J. A. c/ Albadalejo, 40 C - 5. ${ }^{\circ}$ B. 28037, Madrid.

Sugerencia de cita / Suggested citation: Gómez Yáñez, J. A. (2017). La transformación del juego problemático en España. Revista Española de Sociología, 26 (2), 219-252. 


\section{INTRODUCCIÓN}

\section{El concepto de juego problemático}

En 1977, la adicción al juego fue incluida en la International Classification of Disseases (ICD-9) y, en 1980, reconocida como un desorden mental en la tercera revisión del Diagnostic and Statistical Manual (DSM-III) de la Asociación Americana de Psiquiatría, a partir de los trabajos del Dr. Robert Custer. Gossop (1989) sintetizó las características de las conductas adictivas sin sustancia: 1) un deseo compulsivo de llevar a cabo el comportamiento, especialmente cuando no es posible; 2) un déficit para controlar el comienzo, mantenimiento y cierre de la actividad; 3 ) malestar o estado de ánimo alterado cuando la actividad es impedida 0 cesa; 4) persistencia en la conducta pese a la conciencia de que produce efectos negativos al individuo o su entorno.

Rosenthal (1992) y Lesieur y Rosenthal (1998) afinaron la adicción por el juego: 1) una continua 0 intermitente pérdida de control sobre él y sus costes económicos 0 personales; 2) la progresión en la frecuencia, cantidades jugadas y en la preocupación por juego y por obtener dinero para volver a jugar y 3 ) desconocimiento de las consecuencias adversas del abuso, subestimándolas 0 ignorándolas. Se puede añadir pérdida de interés por otras actividades e interferencia en la vida cotidiana (Echeburúa, 1999). En definitiva, la clave de estas adicciones es la pérdida de control del individuo sobre su comportamiento hasta llegar a extremos absurdos, delirantes o peligrosos para él y su entorno, con bloqueo de la conciencia del tiempo y dinero gastado en la actividad, de las perturbaciones sobre su actividad normal y en sus relaciones y de los perjuicios que se pueden derivar (Neal et al., 2005).

Este proceso se puede representar como el ascenso por una pendiente en la que un individuo se siente paulatinamente atraído por una actividad, en este caso, el juego (Figura 1). Operativamente se han establecido cortes para distinguir los niveles de implicación, desde la ausencia u hostilidad hacia el juego —actitud presente en muchos no jugadores - hasta el "alto riesgo" 0 el "juego problemático", es decir, la pérdida de control, que es la fase preclínica o clínica. Sintetizando estos estadios, se podría hablar del jugador social, que juega ocasional o regularmente a juegos populares y legales por entretenimiento, manteniendo el control sobre la actividad, que puede dejarlo cuando desee e incluso olvidarlo. En las fases intermedias el individuo juega con más frecuencia pero el juego no interfiere en sus relaciones sociales, laborales o familiares, sería el estadio de afición. El jugador problemático es el que entra en una dependencia emocional, perdiendo el control a partir de la necesidad de recuperar lo perdido, fracasando en sus intentos de resistir el impulso. Son habituales en esa fase los razonamientos distorsionados sobre la realidad, un optimismo irracional y el pensamiento supersticioso (0choa y Labrador, 1994; Domínguez, 2009). Este este último comportamiento es muy minoritario, aunque es el que más atención recibe en las ciencias sociales.

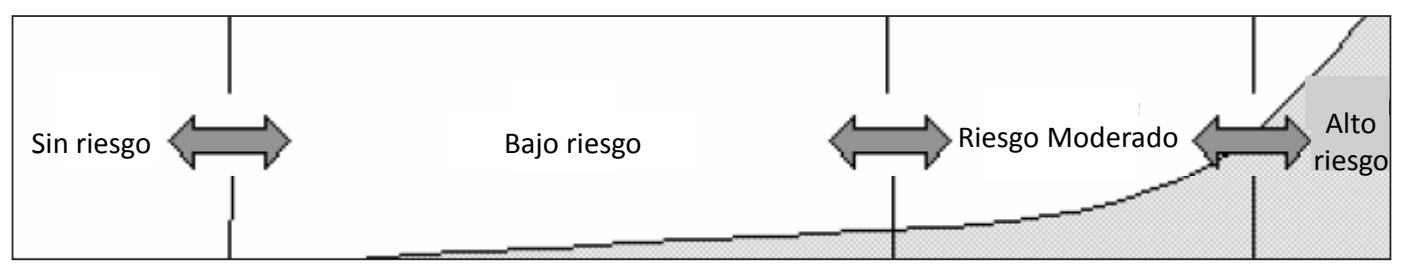

Figura 1. Segmentación de los niveles de riesgo en la adicción al juego

Fuente: Ontario Problem Gambling Research Centre 
Las flechas de doble dirección reflejan que los problemas derivados del juego no son irreversibles ni progresivos. Varios estudios de principios de los 2000 mostraron que la idea de que la atracción por el juego no tiene retorno no se cumple empíricamente, se detectaron altas tasas de recuperación e inversión de la tendencia (Hodgins y El-Guebaly, 2000; Shaffer y Hall, 2002; Slutske et al., 2003; Wiebe et al., 2003; Abbott, Williams y Volberg, 2004).

\section{Los instrumentos de medida y clasificación. Su uso en España}

La investigación clínica utiliza test para diagnosticar los casos individuales, son una herramienta para el psiquiatra o psicólogo, que puede poner sus resultados en relación con otros procedimientos de diagnóstico.

En la investigación sociológica el problema es determinar qué fracción de la población se sitúa en cada corte de continuum "sin riesgo"-"juego problemático". Los primeros estudios sobre el conjunto de la población convirtieron los test clínicos en los cuestionarios estructurados utilizados en encuestas, aplicándolos a muestras representativas. En los ochenta y primera mitad de los noventa se utilizaron los denominados IGB (Inventory of Gambling Behavior) y, posteriormente, el SOGS (South Oaks Gambling Screen), de Lesieur y Blume (1987, 1993, Echeburúa et al., 1994), compuesto por 20 items. Este último se utilizó por primera vez en un estudio en 1986 en el estado de Nueva York (Volberg y Steadman, 1988), desde entonces se convirtió en una herramienta estándar. Hasta mediados de los noventa se hicieron más de 60 estudios con él, incluidos varios en España, lo que facilitó la comparación de resultados.

Con el tiempo, se observó que los resultados de la aplicación del SOGS a muestras representativas de la población no eran precisos. Las revisiones concluyeron que sobreestimaba la población con problemas al recoger falsos positivos (Abbott y Volberg, 1996; Volberg, 1999; Ferris y Wynne, 2001; Lesieur, 2002; Stevens y Young, 2008). Stinchfield (2002) concluyó que casi duplicaba las tasas reales. Una razón de este sesgo es que el cuestionario se refiere a toda la vida del entrevistado lo que produce imprecisiones por fallos de memoria (Dickerson, 1987; Culleton, 1989; Volberg, 1999).

En 1994, la Asociación Americana de Psiquiatría desarrolló el cuestionario DSM-IV (Diagnostic and Statistical Manual of Mental Disorders), del que derivarían varias versiones aplicadas en estudios sociológicos: el DIS (Diagnostic Interview Schudule) (Robins et al., 1981), CIDI (Composite International Diagnostic Instrument) (WHO, OMS, 1990), DIGS (Diagnostic Interview for Gambling Severity) (Winters, Specker y Stinchfield, 1997), etc. La principal de sus variantes fue desarrollada por el North Opinion Research Center de la Universidad de Chicago (NORC), renombrándose como NODS (NORC-DSM-IV Screen for Gambling Problems) (Gernstein et al., 1999). Al reducir los problemas de clasificación dudosa su aplicación supuso una reducción en los niveles de prevalencia registrados por el SOGS (Volberg, 1996, 2002), algo también observado en España.

En 1997, las agencias provinciales canadienses dedicadas a la investigación de problemas sociales plantearon al Canadian Centre of Substance Abuse la necesidad de una metodología para homogeneizar sus estudios sobre el juego. El CCSA desarrolló el CPGI (Canadian Gambling Problem Index) que fue testado en una encuesta dirigida a la población canadiense mayor de edad, validándose sus resultados desde el punto de vista clínico con entrevistas a una submuestra de jugadores problemáticos (Ferris y Wynne, 2001). Consistía en 20 preguntas y entre 2001 y 2005 se utilizó en las provincias de Canadá, con leves retoques (Braid y Volberg, 2008: 8-9), también se utilizó en Australia y Nueva Zelanda.

Fuera de Canadá, una versión sintetizada se adoptó como referente para las investigaciones sociológicas, el PGSI (Problem Gambling Severity Index).

Con el tiempo las investigaciones convergen en la utilización del PGSI/CPGI y el DSM-IV/NODS. A mediados de los noventa se habló de un relativo "caos conceptual y metodológico" (Shaffer, Hall y Van der Bilt, 1997 y 1999), aunque es normal que las discrepancias entre investigadores sociales se mantengan un tiempo en campos científicos 
recién abiertos y eminentemente empíricos, hasta que se imponen ciertas metodologías (Braid y Volberg, 2008, 9; Labrador et al., 2016). Las administraciones públicas y la comunidad científica han tratado de aquilatar sus instrumentos de investigación, tanto en técnicas de campo como en cuestionarios y su explotación estadística, y detectar los sesgos que se producían entre diversos cuestionarios y metodologías. En 2012, Williams, Volberg, Rhis y Stevens analizaron 242 estudios realizados en 202 jurisdicciones para sistematizar y homogeneizar los resultados de las distintas metodologías: el ámbito temporal sobre el que se calculan los niveles de riesgo (considerando el último año 0 toda la vida), los sesgos derivados de los distintos cuestionarios, los ocasionados por la forma de presentar el cuestionario al entrevistado (como un estudio de temática general 0 sobre el juego) y el trabajo de campo (personal, telefónico u online).

Dos aspectos se añaden para producir aquella sensación de "relativo caos metodológico". Primero, la utilización de distintas denominaciones para los niveles de implicación en el juego. En las versiones originales son "descriptivas", pero en algunas investigaciones se traducen de manera que introducen dramatismo e inexorabilidad en un proceso de implicación creciente (en algún estudio español se utilizó "jugadores en progresión adictiva" para los de "riesgo moderado"). En la investigación que se presenta aquí se han mantenido las denominaciones originales y su significado (Tabla 1):

El segundo, es el recelo que desprende el análisis de algunas investigaciones, algo característico en España. Quizá sea por la elevada implicación de algunos investigadores, tendentes a presuponer que los niveles de prevalencia del juego problemático "deberían" ser más elevados (Becoña, 2004a, 181) y que los "nuevos instrumentos de evaluación pueden haber influido en la variación de los datos" (Santos, 2012, 20) 0 a resaltar la existencia de "una confabulación tácita para que el jugador patológico sea ocultado y el juego patológico tratado con apatía" en la que participarían el lobby de empresas del juego y "los gobiernos (que) reciben ingresos" que operarían en un "entramado, actuando como grupo de presión" (Custer, 1989; Salinas, 2004, 36-37; Becoña, 2004b, 27-28). Un trabajo reciente afirma: "En España la tasa de prevaIencia del trastorno de juego/juego problemático puede oscilar entre el $2 \%$ y el $3 \%$ de la población adulta, si bien estas cifras pueden ser algo más bajas cuando la evaluación es más precisa (sic)" (Echeburúa, 2016: 252), el autor es, a su vez, coautor de un estudio realizado en 2013 en el que la tasa de juego problemático entre la población adulta española a lo largo de toda la vida se estima en 1,1\% (Labrador et al., 2013). En suma, el progresivo ajuste de los métodos de investigación ha sido acogido con desconfianza por algunos miembros del mundo académico y de las Administraciones.

En la investigación que aquí se presenta se adoptó una posición ecléctica, similar a la del British Gambling Prevalence Survey 2010 (Wardle et al., 2011), es decir, utilizar los cuestionarios DSM-IV y PGSI conjuntamente, acotados al último año. Esta decisión elude los riesgos de un solo cuestionario y permite una banda de estimaciones sobre la dimensión de cada segmento de sensibilidad ante el juego.

Tabla 1. Segmentación de los niveles de riesgo en la adicción al juego

\begin{tabular}{l|l}
\hline "Recreational Gambler"/“Non problem"/“Sin problema" & No experimenta ninguna consecuencia negativa por jugar \\
\hline "Low risk gambler"/“Sin riesgo" & $\begin{array}{l}\text { Probablemente juega a niveles que no llevan a } \\
\text { consecuencias negativas }\end{array}$ \\
\hline "Moderate risk gambler"/“Riesgo moderado" & $\begin{array}{l}\text { Juega a niveles que pueden o no inducir consecuencias } \\
\text { negativas }\end{array}$ \\
\hline "Problem gambler"/“Jugador problemático" & Juega a niveles que inducen a consecuencias negativas. \\
\hline
\end{tabular}




\section{EL JUEGO PROBLEMÁTICO EN ESPAÑA}

\section{Evolución: descenso y transformación}

En España se han hecho 20 estudios sobre la incidencia del juego problemático en la población adulta. Hay otros sobre poblaciones restringidas (jóvenes, universitarios, mujeres) pero nos ceñiremos a los primeros. 16 son de ámbito autonómico, provincial o local, la Tabla 2 recoge sus características técnicas y los porcentajes de población estimada en las franjas de juego de "riesgo moderado" y de "juego problemático". Se realizaron entre 1990 y 2007. Sobre ellos se han realizado análisis o resúmenes más generales aunque ninguno los contempla todos (Arbinaga, 2000; Append, 2001; Barroso, 2003: 91; Becoña, 2004b: 17; Domínguez Álvarez, 2007 y 2009: 7; Ferrández, 2010: 68). Los cuatro sobre el conjunto del país se hicieron entre 2013 y 2016. En este trabajo se da cuenta de los dos más recientes, recogidos en las ediciones de 2015 y 2016 del estudio Percepción social sobre el juego de azar en España (Gómez Yáñez, Cases Méndez, Gusano Serrano, Lalanda, 2015 y 2016) ${ }^{1}$.

Las tasas de juego problemático entre 1990 y 2000 se situaban entre el $1,7 \%$ y el $2,6 \%$, calculadas habitualmente con cuestionarios SOGS y relativas a la vida del entrevistado. Las más elevadas se localizaron en Cataluña en 1990, 2,4\% (Cayuela, 1991) y 2,3\% (Labrador y Becoña, 1990, pero se trataba de un estudio sobre la

1 Los estudios Percepción social sobre el juego en España se realizan desde 2010, a cargo de un equipo de investigación del Instituto de Política y Gobernanza de la Universidad Carlos III, del que forman parte el profesor Don José Ignacio Cases Méndez, Don Germán Gusano Serrano, Don Carlos Lalanda y el autor; en las ediciones de 2010 y 2011 colaboró Javier Ruiz. Se basan en dos encuestas, una representativa de la población residente en España entre 18 y 75 años ( $n=1000$, margen de error $\pm 3,2 \%$ ), y otra de la población de jugadores online (en 2015, $n=450$, margen de error $\pm 4,7 \%$; en 2016, $n=616$, margen de error $\pm 4,1 \%$ ). En las ediciones de 2015 y 2016 parte del cuestionario se destinó a investigar el juego problemático en ambas poblaciones. La investigación es financiada por la Fundación Codere. población que había jugado), y en las capitales aragonesas, 2,6\% (López Jiménez et al., 1999). Los informes mostraban que había ciudades con tasas más elevadas: Vigo, 3,5\% (Becoña, 1993); Jaén, 2,4\% (Irurita, 1996), aunque pocos años más tarde había descendido al 0,7\% (Salinas, 2002); y Punta Umbría, 3,5\% (Arbinaga, 2000), en la provincia de Huelva, en la que la tasa era $3,8 \%$ (Salinas, 2002). A la vez, había tasas bajas en las grandes ciudades gallegas, menos $\mathrm{A}$ Coruña y Vigo, en 1990, 0,6\% (Becoña, 1993) en 2002, en Córdoba, 0,6\%; y en Granada, 1,1\% (Salinas, 2002). La disparidad de los datos muestra que había significativas diferencias locales en los años noventa.

A partir de 2000 las tasas se redujeron. En Álava se detectó un 0,3\% (Append, 2001) y en Navarra un $0,8 \%$ (Plan Foral de Drogodependencias, 2000). En Galicia, en 2001, Becoña (2004a), utilizando el cuestionario NODS, aplicando un mínimo de $90 €$ de juego anual para incluir a los sujetos en el nivel de juego problemático, detectó un $0,3 \%$ en los últimos doce meses que se ampliaba al $0,9 \%$ a lo largo de la vida. En Cataluña, en 2007, el 0,2\% (López Ibáñez y Volberg, 2007)33.

La tendencia al descenso fue confirmada por los estudios de ámbito nacional realizados entre 2013 y 2016. El de Labrador et al. (2013), utilizando el cuestionario NODS, mostró una tasa de juego problemático del 1,1\% a lo largo de la vida del entrevistado. Williams et al., en el trabajo citado (2012: 22-28), analizaron 54 estudios en los que se aplicaron cuestionarios referidos a toda la vida del entrevistado y al último año, detectando una elevada correlación

2 Ferrol, Lugo, Ourense, Pontevedra y Santiago de Compostela.

3 Los resultados de este estudio llevaron a la Consejería de Interior de la Generalitat de Cataluña a explicar que "la decisión de impulsar un Programa de Juego Responsable no fue una reacción a una situación especialmente problemática sobre las adicciones al juego en Cataluña en relación, comparativamente, con otros países (sic) de nuestro entorno ... no son cifras altas". Generalitat de Cataluña, Departamento de Interior, El Programa de Juego Responsable, 2007-2010, Barcelona, 2010, 16. 


\begin{tabular}{|c|c|c|c|c|c|c|c|c|c|c|c|c|c|c|c|c|c|c|c|c|}
\hline 읕 & & 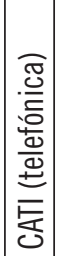 & 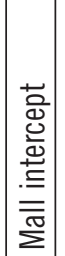 & 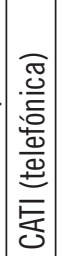 & 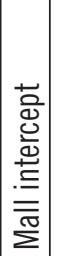 & 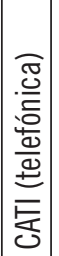 & 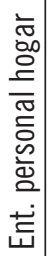 & 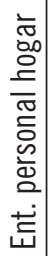 & & 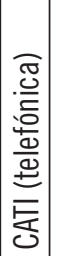 & 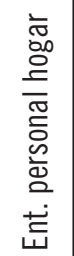 & 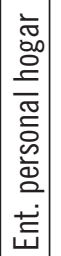 & 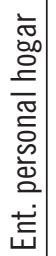 & 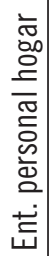 & 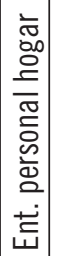 & & 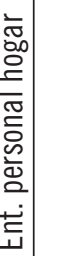 & 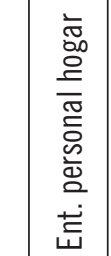 & & 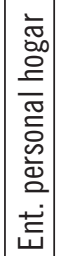 \\
\hline 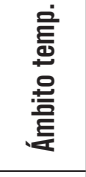 & & 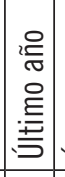 & 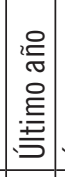 & 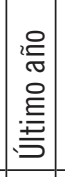 & $\begin{array}{l}\frac{\pi}{0} \\
\frac{0}{3} \\
\frac{0}{0} \\
\frac{\pi}{5} \\
0 \\
\end{array}$ & 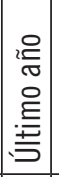 & $\begin{array}{l}\frac{\pi}{0} \\
\frac{0}{0} \\
\frac{\pi}{5} \\
\frac{0}{0}\end{array}$ & 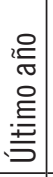 & & 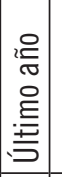 & $\begin{array}{l}\frac{\pi}{7} \\
\frac{\pi}{2} \\
\frac{\pi}{0} \\
\frac{\pi}{0}\end{array}$ & $\begin{array}{l}\frac{\pi}{2} \\
\frac{D}{2} \\
\frac{0}{0} \\
\frac{\pi}{0} \\
\end{array}$ & $\begin{array}{l}\frac{\pi}{7} \\
\frac{0}{2} \\
\frac{0}{\pi} \\
\frac{\pi}{0} \\
\end{array}$ & $\begin{array}{l}\frac{\pi}{7} \\
\frac{\pi}{2} \\
\frac{\pi}{\pi} \\
\frac{\pi}{0}\end{array}$ & $\begin{array}{l}\frac{\pi}{7} \\
\frac{0}{2} \\
\frac{0}{0} \\
\frac{0}{0} \\
1\end{array}$ & & 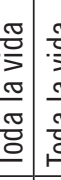 & 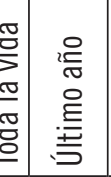 & & \begin{tabular}{|l|}
$\frac{\pi}{0}$ \\
$\frac{0}{3}$ \\
$\frac{0}{0}$ \\
$\frac{\pi}{0}$ \\
0
\end{tabular} \\
\hline 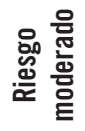 & $\widehat{\varrho}$ & 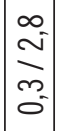 & 0 & $\frac{\tilde{n}}{\tilde{n}}$ & 음 & $\mid$ & $\underset{\leftarrow}{\sim}$ & ma & $\stackrel{0}{-}$ & م' & $\stackrel{\sigma}{\sigma}$ & $\underset{-}{-}$ & छَ. & $\begin{array}{l}\infty \\
\dot{m}^{-}\end{array}$ & $m^{m}$ & & \begin{tabular}{l|l}
0 & r \\
$\sim$ & L
\end{tabular} & $\stackrel{6}{-}$ & & \\
\hline 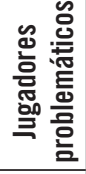 & $\widehat{\varrho}$ & $\mid \begin{array}{l}\overrightarrow{1} \\
\bar{m} \\
0\end{array}$ & $n$ & $\mid \begin{array}{c}5 \\
0 \\
0 \\
0 \\
0\end{array}$ & $\exists$ & $\tilde{\sigma}$ & $\Rightarrow$ & m. & $\stackrel{\infty}{\infty}$ & $m$ & $\stackrel{\rho}{m}$ & $\stackrel{0}{-}$ & $\stackrel{0}{\sim}$ & $\stackrel{s}{\rightarrow}$ & $\Rightarrow$ & $\stackrel{\circ}{\sim}$ & $\because 7$ & $\hat{\Rightarrow}$ & $\stackrel{m}{\sim}$ & $\stackrel{\sim}{\sim}$ \\
\hline 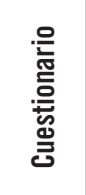 & & $\begin{array}{l}\geq \\
\sum \\
\bar{D} \\
\overline{0} \\
\bar{c} \\
\vdots\end{array}$ & 연 & $\begin{array}{l}\geq \\
\sum \\
0 \\
\bar{D} \\
\bar{D} \\
0\end{array}$ & 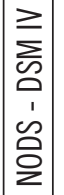 & ळ্ & $\begin{array}{l}\text { s } \\
\text { ऽ }\end{array}$ & 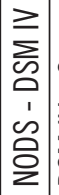 & 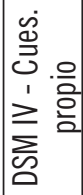 & $\begin{array}{l}\text { लs } \\
\text { कू } \\
\text { क }\end{array}$ & స్ & $\begin{array}{l}\text { Dु } \\
\text { क् }\end{array}$ & $\begin{array}{l}\text { क् } \\
\text { के }\end{array}$ & 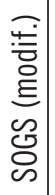 & 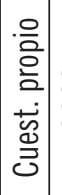 & \begin{tabular}{l|l} 
Dु \\
Oூ
\end{tabular} & 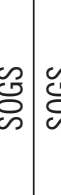 & 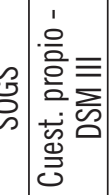 & & $\begin{array}{l}\text { Sु } \\
\text { D }\end{array}$ \\
\hline ఏ & & $\underset{\Xi}{\Xi}$ & $\underset{\Xi}{\beth}$ & ఠิ & 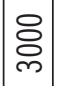 & 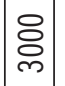 & 官 & $\mid$ & 吉 & $\begin{array}{l}\infty \\
\Xi \\
\Xi\end{array}$ & 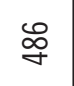 & ষ্ৰి & ৪ి & $\stackrel{\sigma}{\vec{g}}$ & 今ิ & & 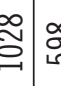 & $\stackrel{0}{\infty}$ & $\widehat{\omega}$ & ஜ \\
\hline 量 & & 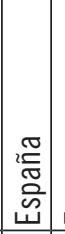 & 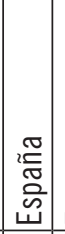 & 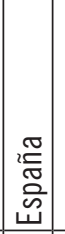 & 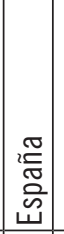 & 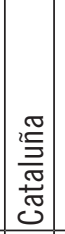 & 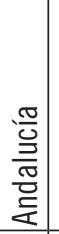 & 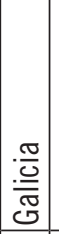 & 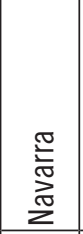 & : & 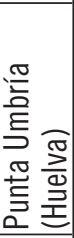 & \begin{tabular}{|l|} 
\\
$\frac{0}{0}$ \\
$\frac{3}{0}$ \\
$\frac{7}{0}$ \\
$\frac{0}{\frac{1}{2}}$ \\
\end{tabular} & 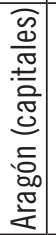 & 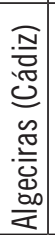 & $\mid \begin{array}{l}\frac{0}{U} \\
\frac{\mathrm{O}}{\mathrm{T}} \\
\frac{\mathrm{O}}{\mathrm{C}} \\
\end{array}$ & 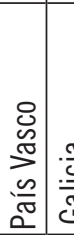 & 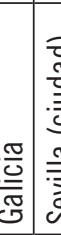 & 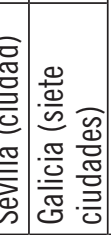 & 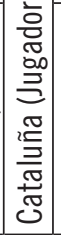 & 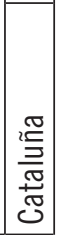 \\
\hline 选 & & 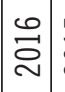 & 足 & 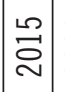 & 豙 & 용 & శ్రి & 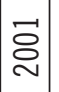 & 各 & 各 & 穴 & 穴 & 官 & $\begin{array}{l}\infty \\
\stackrel{\circ}{二}\end{array}$ & 志 & ஜூ & F & 察 & 亩 & 욤 \\
\hline 产 & & 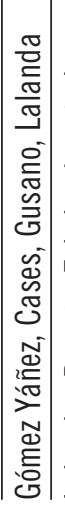 & 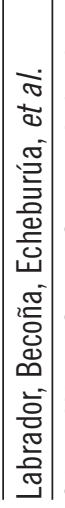 & 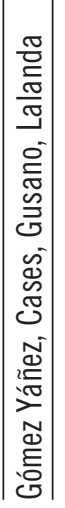 & 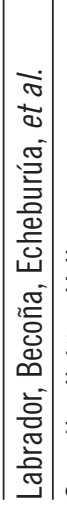 & 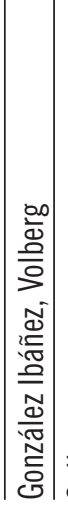 & 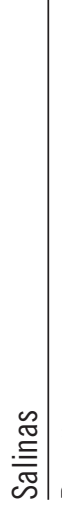 & 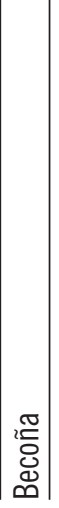 & 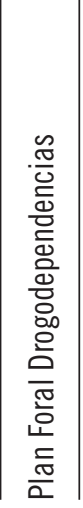 & $\left|\begin{array}{|c|} \\
\frac{0}{0} \\
\frac{0}{0} \\
\frac{a}{2}\end{array}\right|$ & 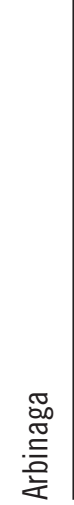 & 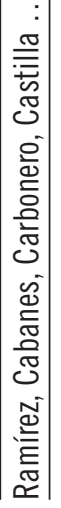 & 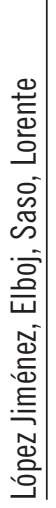 & : & 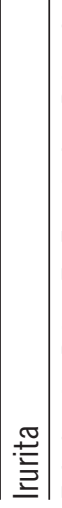 & 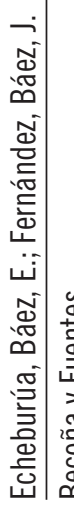 & 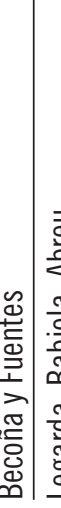 & 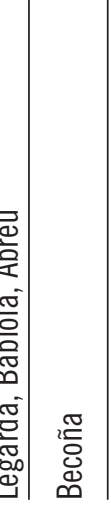 & 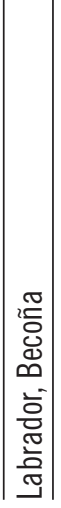 & $\mid$ \\
\hline
\end{tabular}


entre ambos datos $\left(r^{2}=0,920\right)$, estimaron que para "convertir" el dato de toda la vida al último año, que se debería considerar la medida estándar de este tipo de estudios, hay que multiplicar el primero por 0,44. Por tanto, en este estudio, la tasa ajustada al último año se situaría en 0,48\% para el juego problemático, muy coherente con los estudios posteriores. Este estudio tiene otra peculiaridad que lo separa de las metodologías normalmente utilizadas (entrevistas personales, telefónicas u online en el hogar del entrevistado): su trabajo de campo se hizo por mall intercept, es decir, localizando y entrevistando a los encuestados en la calle, con una media de 16 entrevistas por punto de contacto/día, con control de cuotas de sexo y edad ${ }^{4}$. En 2015, los mismos autores, en un estudio para la Dirección General de Ordenación del Juego, situaban la prevalencia del (NODS) en el $0,3 \%$ en el último año y el $0,9 \%$ a lo largo de toda la vida. En este estudio también se utilizó el sistema mall intercept para captar a los entrevistados, que se describe así en el informe: "la encuesta presencial realizada mediante interacción fuera del hogar del encuestado con un entrevistador entrenado al efecto" (o sea, "a pie de calle", se aclaró en la presentación) (Labrador y otros, 2016, 17). Como es conocido, este sistema rompe la sistemática del muestreo probabilístico, "contemplado desde una óptica estricta de teoría del muestreo, este método de reclutamiento puede generar muestras con importantes déficits de representatividad ... quienes transitan por un lugar constituyen una muestra sesgada de la población" (Sendín, 20-21).

Los datos anteriores son similares a los obtenidos en nuestra investigación en 2015 y 2016, con Ios cuestionarios PGSI y DSM-IV, con entrevistas telefónicas, en los que las tasas de juego problemático quedaban entre $0,1 \%$ y $0,4 \%$ utilizando el cuestionario DSM-IV y $0,1 \%$ y $0,3 \%$ con el PGSI, respectivamente, según el año.

De los estudios realizados en España se extraen varias conclusiones.

4 Información facilitada por el director del estudio, Labrador, en su presentación en el Consejo de Juego Responsable de la DGOJ, Madrid, 2014.
Hay una tendencia al descenso del juego problemático destacada por algunos autores con renuencia: "Los estudios de prevalencia realizados en muestras representativas de la población basados en el DSM-III, DSM-III-R, DSM-IV y SOGS indicaban una prevalencia entre el $1,4 \%$ y el $1,7 \%$... semejante a la de otros países. Los últimos estudios con instrumentos derivados del DSM-IV, como el NODS, o versiones derivadas del SOGS, indican un nivel de prevalencia menor para el juego patológico" (Becoña, 2004b: 9). En 2012, el Manual de Intervención en Juego Patológico de Extremadura, de la Secretaría Técnica de Drogodependencias de la Junta de Extremadura, a cargo de un equipo de 13 responsables de centros de atención públicos, altos cargos de la administración autonómica y profesores de psicología, señalaba: "si hace unos años, tanto los datos nacionales (no se citan las fuentes, no tenemos localizado ningún estudio nacional anterior a 2012) como de otros países, indicaban que entre el $1,5 \%$ y el $2 \%$ de la población era jugador patológico, los últimos estudios apuntan a un porcentaje entre el 0,3 y el 0,9 de jugadores patológicos, siendo un poco mayor para los jugadores problemas (sic) ... (pese a lo anterior) ... La mayor parte de los autores consideran que se debe continuar investigando para aproximarse a los datos reales de la epidemiología del juego patológico" (Santos, 2012).

Como se vio, en los noventa había diferencias geográficas significativas. Había elevadas tasas de juego problemático en algunas ciudades que tenían problemas de desmantelamiento del tejido productivo industrial (Vigo, Algeciras, Punta Umbría y parte de Huelva, las ciudades de Cádiz y Málaga, Irurita, 1994) mientras que en otras este problema estaba muy poco extendido.

El juego problemático se ha reducido sensiblemente en España en los últimos 25 años. Aunque convertir los datos de estudios heterogéneos y de ámbitos territoriales diferentes en una figura es heterodoxo, de la Figura 2 se deduce el descenso de la dimensión problema. La línea discontinua muestra la tendencia de los datos originales, la continua la tendencia ajustada al "último año", según los factores de conversión de Williams, Volberg y Stevens, 2012. 


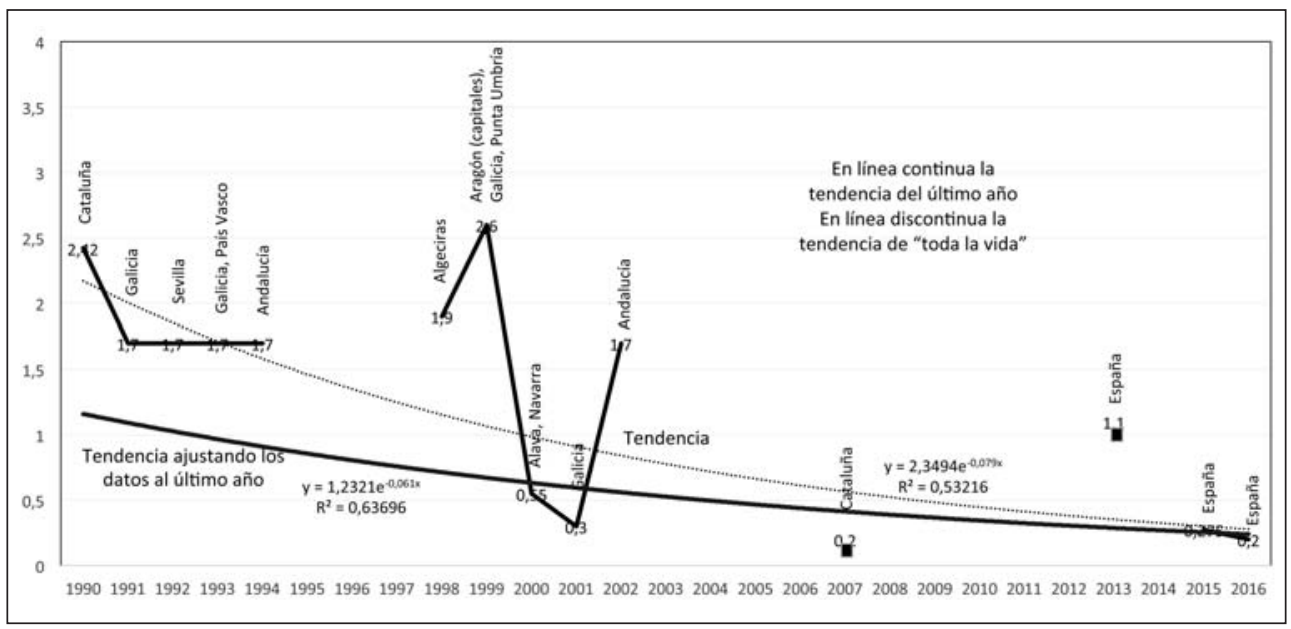

Figura 2. Evolución de la incidencia del juego problemático en la población adulta residente en España (1990-2016)

Fuente: Elaboración propia a partir de los estudios recogidos en la Tabla 2

Porcentajes inferiores al 0,5\% de la población, como los que se registran en la última década y media, sitúan el problema en detectar conductas individuales de riesgo más que perfiles sociológicos. Es decir, hay unas características sociológicas, demasiado amplias, que parecen facilitar que surja un problema, pero no se puede decir que afecte a ciertos sectores sociales concretos en una proporción significativa. Tasas tan reducidas sólo pueden significar que los factores individuales influyen mucho, es posible que permanezcan latentes en algunos sujetos y se activen por causas ambientales, pero esto reconduce el problema a características de la personalidad, factores biológicos, de aprendizaje, déficit cognitivos, etc. (Domínguez, 2009: 9-16), es decir, al ámbito de la psiquiatría en el que está abierto un debate teórico sobre si el juego problemático puede clasificarse como una adicción o como un heterogéneo trastorno con componentes obsesivocompulsivos y adictivos (Blanco et al., 2001; Moreyra et al., 2002; Rotgers y Christner, 2005; Reilly y Smith, 2013) o de descontrol de los impulsos (Potenza y Hollander, 2002), con afinidades con las compras impulsivas (Black et al., 2010), en el que la herencia genética tiene influencia (Potenza et al., 2005) así como los factores familiares que vendrían a explicar entre el $35 \%$ y el $55 \%$ de los síntomas que definen el juego patológico (Eisen et al., 1998), los aspectos neurobiológicos individuales (Saiz e Ibáñez, 1999), 0 la búsqueda de sensaciones fuertes y emotividad (Jiménez-Murcia et al., 2012) que están presentes en otros trastornos adictivos o conductas atípicas.

El perfil de los jugadores problemáticos en España ha cambiado. En los años noventa eran mayoritariamente hombres (en proporción de dos tercios 0 tres cuartos sobre las mujeres, en algún estudio todos los afectados eran hombres), de edades intermedias y jóvenes, niveles culturales medios 0 bajos, atraídos por las máquinas tragamonedas. Era el patrón común, repetido en otros países, que se complementaba con poli-adicciones en una proporción relevante de los casos como el alcohol (Becoña, 2004: 15), y el tabaco (Irurita, 1996; Arbinaga, 2000). Stinchfield y Winters (1998) y Costes et al. (2011), en Estados Unidos y Francia, respectivamente, identificaron factores comunes en las adicciones al alcohol y al juego $0^{5}$; tam-

5 Suecia (Rönnberg et al., 1999), Suiza (Bondolfi et al., 2008), Sudáfrica (Collins y Barr, 2003), Estonia (Laansoo y Niit, 2009), Florida (Shapira et al., 2001), Delaware (School of Urban Affairs, 2002), Victoria (McMillen et al., 2004), lowa (Gonnerman y Lutz, 2011). 
bién con el tabaco ${ }^{6}$. En Canadá se ha encontrado que el consumo de drogas suele ser más alto entre los jugadores problemáticos 0 de riesgo moderado (MarketQuest Research, 2009: 65). La evidencia empírica sugiere que el juego problemático, en ocasiones, forma parte de una compleja sintomatología poliadictiva y de trastornos de la personalidad como depresión, dificultades en las relaciones personales y sociales, etc. (Echeburúa, 2016; 252).

Los estudios más recientes, de 2015 y 2016 (Gómez Yáñez et al.) muestran un perfil distinto: mayoritariamente hombres jóvenes, se mantiene la reducida presencia de mujeres, mayoritariamente menores de 35 años, con una elevada proporción de personas que juegan online, aunque se mantiene la incidencia de máquinas tragamonedas, de niveles educativos medios 0 altos y de todos los status sociales, aunque con cierta inclinación hacia los medio altos y altos. En conclusión, el perfil ha cambiado aunque permanece que afecta mayoritariamente a los hombres rasgo común en todos los países (estos estudios no han analizado la eventual poliadicción). Esto coincide con la información procedente de los centros públicos de atención 0 de asociaciones de afectados: las personas que están en tratamiento 0 acuden a estos centros son muy jóvenes, menores de 30 años en algunos casos adolescentes, atraídos por el juego online, junto a ellos hay otro segmento de edades intermedias y adicción más habitual hacia las tragamonedas ${ }^{7}$. En otros países también se ha encontrado una elevada correlación entre el juego online y el juego problemático: Gran Bretaña (Wardle et al., 2011), Irlanda del Norte (2010).

¿Por qué se ha producido este descenso y transformación en el juego problemático? Desde luego, por un incremento en la oferta de ocio en el último cuarto de siglo, también, plausiblemente, por medidas como la prohibición de fumar en locales cerrados. Por otro lado, el incremento de

6 Nueva Zelanda (Mason, 2009), Canadá (Wood y Williams, 2009), Gran Bretaña (Wardle et al., 2010), Francia (Costes et al., 2011), Florida (Shapira et al., 2001), Alberta (Williams et al., 2011), lowa (Gonnermann y Lutz, 2011).

7 Véanse las fuentes en la Tabla 4. locales de juego no correlaciona linealmente con el juego problemático, pese a lo que se dice convencionalmente, después del impacto de la novedad de un producto (las máquinas tragamonedas en los ochenta, el juego online en los 2010) los individuos y la sociedad se adaptan y aprenden los riesgos que producen (Abbott, 2006). Es decir, toda oferta de entretenimiento tiene un impacto inicial que posteriormente se normaliza, en la fase inicial, hay sistemáticamente un porcentaje de población que sobrepasa las dosis razonables (LaPlante y Shaffer, 2007). Por tanto, "el problema de las políticas públicas es cuánto puede tardar la adaptación y cuándo se puede prever que un grupo expuesto a nueva atracción aprenda a dosificarla" (Shaffer, 2005: 1228), lo que ahora se plantea con el juego online.

\section{El juego problemático en España 2015/16}

\section{Metodología}

En las ediciones de 2015 y 2016 del estudio Percepción social sobre el juego de azar en España VI y VII (Gómez Yáñez et al., 2015 y 2016) se incluyeron los cuestionarios PGSI y DSM-IV. Las muestras, representativas de la población residente en España entre 18 y 75 años, tenían una dimensión de 1002 y 1012 individuos, respectivamente, las preguntas se ciñeron a los últimos doce meses, la temática del cuestionario versaba sobre la práctica del juego, pero al comienzo se preguntaba por diversos consumos para evitar rechazos atribuibles a la temática (ver Anexo I).

\section{Resultados}

Se puede concluir que el juego problemático en España afecta a entre el $0,1 \%$ y el $0,3 \%$ de la población entre 18 y 75 años (Tabla 3), es decir, entre 34200 y 102000 individuos. En "riesgo moderado" se sitúa entre el $0,3 \%$ y el 3,2\%, tomando los datos extremos de los dos años. En otro estudio de 2015 (Labrador et al., 2016: 29) la tasa de juego problemático fue $0,3 \%$ y la de riesgo moderado $0,6 \%$. 
Tabla 3. Incidencia del juego problemático en España (18-75 años) (\%)

\begin{tabular}{lcccccc}
\hline & \multicolumn{5}{c}{ Metodología de estimación } \\
\hline & \multicolumn{7}{c}{ PGSI } & \multicolumn{5}{c}{ DSM. IV } \\
\hline & 2015 & 2016 & 2015 & 2016 & 2015 & 2016 \\
\hline No jugadores & 18,1 & 16,5 & 18,1 & 16,5 & 18,1 & 16,5 \\
\hline Sin problema & 78,5 & 80,5 & 68,1 & 73,7 & 79,9 & 81,9 \\
\hline Bajo riesgo & 2,6 & 2,4 & 10,2 & 6,9 & - & - \\
\hline Biesgo moderado & 0,7 & 0,3 & 3,2 & 2,8 & 1,9 & 1,4 \\
\hline Problemático & 0,1 & 0,3 & 0,4 & 0,1 & 0,1 & 0,2 \\
\hline (n) & $(1002)$ & $(1012)$ & $(1002)$ & $(1012)$ & $(1002)$ & $(1012)$ \\
\hline
\end{tabular}

Fuente: Gómez Yáñez, J. A., Cases, J. I., Gusano, G., Lalanda, C. (2015 y 2016)

Tabla 4. Datos parciales sobre personas atendidas o en tratamiento por juego problemático por Servicios Públicos de Salud o Asociaciones de Afectados

\begin{tabular}{l|l|l|l|l|r}
\hline \multicolumn{1}{c|}{ Concepto } & \multicolumn{1}{c|}{ Fuente } & Ámbito de referencia & \multicolumn{1}{c|}{2013} & 2014 & 2015 \\
\hline Individuos atendidos & Asociaciones de FEJAR $\left(^{*}\right)$ & España & 13625 & 15500 & 14735 \\
\hline Individuos atendidos & Aralar (Navarra) $\left(^{*}\right)$ & Navarra & & & 207 \\
\hline Individuos atendidos & Agalure (A Coruña) $\left(^{*}\right)$ & Galicia & & & 102 \\
\hline Pacientes en tratamiento & Junta de Andalucía (9 centros) & Andalucía & 2527 & 2719 & \\
\hline Individuos y familiares atendidos & Indalajer (Almería) (*) & Almería (Andalucía) & & & 150 \\
\hline Pacientes en tratamiento (al año) & Hospital Bellvitge (Barcelona) & Unidad de tratamiento & & & 350 \\
\hline Pacientes en tratamiento & Serv. Salud Asturias (SESPA) & Asturias & 130 & & \\
\hline Pacientes en tratamiento & Serv. Atención a Ludopatía & $\begin{array}{l}\text { Santa Cruz Tenerife } \\
\text { (Prov.) }\end{array}$ & 70 & & \\
\hline
\end{tabular}

(*) FEJAR: Federación Española de Jugadores de Azar Rehabilitados.

Fuentes: Fejar: El País, Madrid, 24-03-2016. Aralar: Diario de Noticias, Pamplona, 17-02-2016. Agalure: La Opinión, A Coruña, 02-02-2017. Junta de Andalucía, Consejería de Igualdad, Salud y Políticas Sociales, Europa Press, 28-10-2013; http://www. elmundo.es/andalucia/2013/10/28/526e46310ab740ed2d8b457e.html; Diario de Sevilla, Sevilla, 11-11-2014. Indalajer: La Voz de Almería, 12-12-2016. Cataluña, Bellvitge: Ara, Barcelona, 01-04-2016. Asturias: La Voz de Asturias, 4-10-2016. lavozdeasturias.es. Santa Cruz de Tenerife: Castro, Jesús: http://juegopatologico.blogspot.com.es/2007/09/algunos-datosestadsticos-de-personas.html 
No hay datos oficiales sobre el número de personas en tratamiento por trastornos derivados del juego. Los disponibles proceden de las asociaciones de afectados y servicios de salud (Tabla 4). La Federación Española de Jugadores de Azar Rehabilitados (FEJAR), que integra a 25 asociaciones, informa sobre el número de sus socios "afectados por la ludopatía y otras adicciones conductuales", 15500 personas.

\section{Estimación del juego problemático con el cuestionario PGSI}

Consiste en nueve ítems. En su versión original se requerían respuestas dicotómicas (sí/no), pero en versiones posteriores se planteó como escala de frecuencia (Likert) de cuatro puntos ${ }^{8}$, asignando a cada respuesta una puntuación, de 0 a 3 , siendo 0 la respuesta "nunca" hasta 3 "casi siempre", así se sitúa a cada entrevistado en un punto entre 0 y 27 (9 preguntas por una puntuación máxima de 3) al sumar los valores de sus respuestas se clasifica en uno de los cuatro niveles:
$\checkmark 0$ "Sin problema"
$\checkmark 1$ y 2 "Jugador $\sin$ riesgo"
$\checkmark 3$ a 7 "Jugador en riesgo moderado"
$\checkmark 8$ y más "Jugador problemático"

La Tabla 5 recoge los resultados a la muestra total de los trabajos de campo realizados en enero de 2015 y 2016 (2014 individuos).

Tabla 5. Respuestas a los ítems del cuestionario PGSI (2015-16) (\%) ( $n=2.014)$

\begin{tabular}{l|c|c|c|c|c|}
\hline & $\begin{array}{c}\text { Casi } \\
\text { siempre }\end{array}$ & $\begin{array}{c}\text { Con } \\
\text { frecuencia }\end{array}$ & $\begin{array}{c}\text { Alguna } \\
\text { vez }\end{array}$ & Nunca & $\begin{array}{c}\text { No } \\
\text { jugador }\end{array}$ \\
\hline En los últimos doce meses ...: & & & & & \\
\hline ¿Ha vuelto para intentar ganar el dinero que perdió? & 0,1 & 0,1 & 1,5 & 80,9 & 17,3 \\
\hline $\begin{array}{l}\text { ¿Ha jugado o apostado más dinero del que se podía } \\
\text { permitir perder? }\end{array}$ & 0,1 & 0,0 & 0,8 & 81,7 & 17,3 \\
\hline $\begin{array}{l}\text { ¿Ha habido gente que le ha criticado lo que juega, } 0 \\
\text { le ha dicho que tiene un problema con el juego, con } \\
\text { independencia de que usted piense que es verdad o no? }\end{array}$ & 0,0 & 0,0 & 0,7 & 82,0 & 17,3 \\
\hline $\begin{array}{l}\text { ¿Ha sentido que el juego le ha provocado problemas } \\
\text { económicos a usted 0 a su familia? }\end{array}$ & 0,1 & 0,0 & 0,3 & 82,3 & 17,3 \\
\hline $\begin{array}{l}\text { ¿Se ha sentido culpable sobre la forma en que juega y lo } \\
\text { que sucede cuando juega? }\end{array}$ & 0,0 & 0,1 & 0,2 & 82,3 & 17,3 \\
\hline $\begin{array}{l}\text { ¿Ha necesitado jugar más dinero para conseguir la } \\
\text { misma emoción? }\end{array}$ & 0,0 & 0,0 & 0,2 & 82,4 & 17,3 \\
\hline $\begin{array}{l}\text { ¿Ha sentido que usted podía tener un problema con el } \\
\text { juego? }\end{array}$ & 0,1 & 0,0 & 0,2 & 82,3 & 17,3 \\
\hline $\begin{array}{l}\text { ¿Ha tomado dinero prestado o vendido algo para } \\
\text { conseguir dinero para jugar? }\end{array}$ & 0,0 & 0,0 & 0,1 & 82,5 & 17,3 \\
\hline $\begin{array}{l}\text { ¿Ha sentido que el juego le ha producido problemas de } \\
\text { salud, incluyendo estrés 0 ansiedad? }\end{array}$ & 0,0 & 0,0 & 0,0 & 82,6 & 17,3 \\
\hline
\end{tabular}

Fuente: Gómez Yáñez, J. A., Cases, J. I., Gusano, G., Lalanda, C. (2015 y 2016)

8 En Australia se empleó una escala de cinco puntos entre 2004 y 2008 (Sproston et al., 2012). 
Tabla 6. Puntuaciones del cuestionario PGSI en España, Gran Bretaña y Australia del Sur

\begin{tabular}{|c|c|c|c|c|}
\hline Nivel de riesgo & Puntuación & España (2015-16) & Gran Bretaña (2010) & South Australia (2012) \\
\hline \multirow{2}{*}{ Sin riesgo } & No jugadores & 17,28 & & 31,2 \\
\hline & 0 & 79,54 & 91,9 & 58,6 \\
\hline \multirow{2}{*}{ Bajo riesgo } & 1 & 2,04 & 3,9 & 5,4 \\
\hline & 2 & 0,50 & 1,7 & 1,7 \\
\hline \multirow{5}{*}{ Riesgo moderado } & 3 & 0,35 & 0,7 & 1,1 \\
\hline & 4 & 0,00 & 0,4 & 0,5 \\
\hline & 5 & 0,10 & 0,4 & 0,4 \\
\hline & 6 & 0,05 & 0,4 & 0,2 \\
\hline & 7 & 0,00 & 0,2 & 0,2 \\
\hline \multirow{2}{*}{ Jugador problemático } & 8 a 15 & 0,10 & 0,2 & 0,6 \\
\hline & 16 a 27 & 0,05 & 0,2 & \\
\hline (n) & & (2014) & (7747) & $(6000)$ \\
\hline
\end{tabular}

Fuentes: España: Gómez Yáñez, J. A. et al. (2015-16). Gran Bretaña: Wardle, H. et al. (2011). Australia del Sur: Department for Communities and Social Inclusion (2013)

El desglose de las puntuaciones de los entrevistados con este cuestionario en España es muy similar a Gran Bretaña (2010) y a Australia del Sur (2012), no hay diferencias relevantes (Tabla 6).

Las mayores tasas de juego problemático y de riesgo moderado se localizan entre los menores de 25 años: $1,2 \%$ y $2,4 \%$, respectivamente. El juego problemático también está por encima de la media entre los 25 y 34 años (0,6\%). Pero, para contrapesar esto, los jóvenes de 18 a 25 años son los que menos juegan en España. Por tanto, se trata de un problema de jóvenes, no necesariamente generacional. El status social incide de forma confusa: hay tasas elevadas en los status alto y medio alto, y entre quienes se pueden ubicar en el bajo, pero menos entre los intermedios. La idea de fondo es que incluso en los sectores en los que se detectan más casos, la incidencia del juego problemático es muy limitada (Tabla 7).

Dos elementos relevantes, la tasa de juego problemático es mayor entre quienes declaran jugar con frecuencia diaria o mayor que semanal. En los hogares con problemas económicos no hay incidencia del juego problemático lo que anula la idea de que las urgencias económicas induzcan al juego como vía de escape 0 de resolución del problema.
Como se señalaba antes, la cultura del juego en España, basada en una larga tradición, está muy dominada por la seguridad de que, a la larga, se pierde, salvo golpe de suerte; por tanto, estos hogares no gastan dinero en juego.

\section{Estimación del juego problemático con el cuestionario DSM-IV}

En su versión original, consiste en 10 preguntas dicotómicas, la suma de respuestas positivas sitúa al entrevistado en tres niveles:
$\checkmark 0$ "sin problema"
$\checkmark 1$ - 2 "riesgo moderado"
$\checkmark 30$ más "juego problemático"
Una versión posterior en escala Likert asigna estrato correspondiente:
$\checkmark 0$
"sin problema"
$\checkmark 1-2$
"bajo riesgo"
$\checkmark 3-7$
"riesgo moderado"
$\checkmark 80$ más
"jugador problemático" una puntuación a cada respuesta: casi siempre (3), con frecuencia (2), alguna vez (1), nunca (0). Sumando las respuestas de cada entrevistado resulta una puntuación entre 0 y 30 que lo sitúa en el 
A esta explotación se le denomina "Continuous Scoring" y es utilizada en el British Gambling Prevalence Survey (Wardle et al., 2011). La versión posterior, DSM-V, ha sido poco utilizada hasta ahora (Reilly y Smith, 2013).
El desglose de las respuestas a las preguntas se recoge en la Tabla 8. La aplicación de los dos sistemas de tabulación descritos lleva a datos levemente diferentes, pero a conclusiones similares.

Tabla 7. Perfil sociológico por niveles de riesgo según el cuestionario PGSI (2015-16) (\%)

\begin{tabular}{l|c|c|c|c|c|c}
\hline & Problemáticos & Riesgo Moderado & Bajo Riesgo & Sin problema & No jugadores & (n) \\
\hline Total & 0,2 & 0,5 & 2,5 & 79,5 & 17,3 & $(2014)$ \\
\hline Sexo: & & & & & & \\
\hline Hombres & 0,3 & 0,8 & 3,5 & 78,1 & 17,3 & $(1006)$ \\
\hline Mujeres & 0,1 & 0,2 & 1,6 & 80,9 & 17,3 & $(1008)$ \\
\hline Edad: & & & & & & \\
\hline De 18 a 24 años & 1,2 & 2,4 & 4,1 & 57,1 & 35,3 & $(170)$ \\
\hline De 25 a 34 años & 0,6 & 0,3 & 2,8 & 75,4 & 21,0 & $(353)$ \\
\hline De 35 a 44 años & 0,0 & 0,4 & 3,1 & 81,3 & 15,3 & $(523)$ \\
\hline De 45 a 54 años & 0,0 & 0,2 & 2,1 & 84,0 & 13,6 & $(419)$ \\
\hline De 55 a 64 años & 0,0 & 0,4 & 1,9 & 85,7 & 12,0 & $(259)$ \\
\hline De 65 a 75 años & 0,0 & 0,3 & 1,4 & 82,4 & 15,9 & $(290)$ \\
\hline Status Social: & & & & & & \\
\hline Status alto & 0,4 & 0,0 & 1,6 & 84,7 & 13,3 & $(255)$ \\
\hline Status medio-alto & 0,3 & 0,7 & 4,3 & 79,8 & 14,9 & $(302)$ \\
\hline Status medio-medio & 0,1 & 0,8 & 1,6 & 79,7 & 17,8 & $(752)$ \\
\hline Status medio-bajo & 0,0 & 0,3 & 3,6 & 77,2 & 18,9 & $(338)$ \\
\hline Status bajo & 0,3 & 0,3 & 2,7 & 77,4 & 19,3 & $(367)$ \\
\hline
\end{tabular}

Tipología de jugador según frecuencia de juego declarada

\begin{tabular}{l|c|c|c|c|c|c}
\hline Cotidianos & 1,1 & 0,0 & 6,9 & 92,0 & 0,0 & $(87)$ \\
\hline Semanales & 0,3 & 1,0 & 4,3 & 94,5 & 0,0 & $(400)$ \\
\hline Esporádicos & 0,0 & 1,2 & 2,8 & 96,0 & 0,0 & $(251)$ \\
\hline Infrecuentes & 0,1 & 0,2 & 2,1 & 97,5 & 0,0 & $(845)$ \\
\hline Se consideran "no jugadores" & 0,0 & 0,2 & 0,0 & 18,3 & 81,5 & $(427)$ \\
\hline
\end{tabular}

Personas que viven en hogares con retrasos en:

\begin{tabular}{l|c|c|c|c|c|c}
\hline -Pago de hipotecas & 0,0 & 0,0 & 9,1 & 65,9 & 25,0 & $(44)$ \\
\hline -Pago de compras & 0,0 & 0,0 & 4,0 & 84,0 & 12,0 & $(25)$ \\
\hline -Pago de servicios/suministros & 0,0 & 0,0 & 12,1 & 75,9 & 12,1 & $(58)$ \\
\hline
\end{tabular}

Fuente: Gómez Yáñez, J. A., Cases, J. I., Gusano, G., Lalanda, C. (2015 y 2016) 
Tabla 8. Respuestas a los ítems del cuestionario DSM-IV (2015-16) (\%) ( $\mathrm{n}=2014)$

\begin{tabular}{|c|c|c|c|c|c|}
\hline En los últimos doce meses: & $\begin{array}{l}\text { Cada vez } \\
\text { que pierdo }\end{array}$ & $\begin{array}{l}\text { La mayor parte } \\
\text { de las veces }\end{array}$ & $\begin{array}{l}\text { Más o menos la } \\
\text { mitad de las veces }\end{array}$ & Nunca & $\begin{array}{c}\text { No } \\
\text { jugador }\end{array}$ \\
\hline \multirow{2}{*}{$\begin{array}{l}\text { Cuando usted juega., ¿con qué } \\
\text { frecuencia vuelve a jugar para ganar lo } \\
\text { que perdió otro día? }\end{array}$} & 0,8 & 1,1 & 6,5 & 74,3 & 17,3 \\
\hline & $\begin{array}{l}\text { Con } \\
\text { frecuencia }\end{array}$ & $\begin{array}{l}\text { Algunas } \\
\text { veces }\end{array}$ & Ocasionalmente & Nunca & $\begin{array}{l}\text { No } \\
\text { jugador }\end{array}$ \\
\hline $\begin{array}{l}\text { ¿Con cuanta frecuencia se ha } \\
\text { encontrado usted mismo pensando } \\
\text { sobre el juego, es decir, volviendo sobre } \\
\text { partidas anteriores, planificando la } \\
\text { próxima vez que jugará o pensando } \\
\text { formas para tener dinero para jugar? }\end{array}$ & 0,6 & 1,9 & 2,4 & 77,9 & 17,3 \\
\hline $\begin{array}{l}\text { ¿Tiene necesidad de jugar más y más } \\
\text { dinero para conseguir la emoción que } \\
\text { desea conseguir jugando? }\end{array}$ & 0,0 & 0,4 & 0,4 & 81,9 & 17,3 \\
\hline $\begin{array}{l}\text { ¿Se siente usted irritable cuando } \\
\text { intenta dejar de jugar? }\end{array}$ & 0,0 & 0,3 & 0,2 & 82,1 & 17,3 \\
\hline $\begin{array}{l}\text { ¿Ha jugado para escapar de sus } \\
\text { problemas o cuando está deprimido, } \\
\text { ansioso o mal consigo mismo? }\end{array}$ & 0,0 & 0,4 & 0,4 & 81,8 & 17,3 \\
\hline $\begin{array}{l}\text { ¿Ha mentido a su familia } 0 \text { a otros } \\
\text { para esconder el tiempo que dedica a } \\
\text { jugar? }\end{array}$ & 0,0 & 0,1 & 0,2 & 82,3 & 17,3 \\
\hline $\begin{array}{l}\text { ¿Ha intentado controlar o recortar lo } \\
\text { que juega o dejar de jugar? }\end{array}$ & 0,2 & 0,7 & 0,7 & 81,0 & 17,3 \\
\hline $\begin{array}{l}\text { ¿Ha cometido una falta } 0 \text { un delito } \\
\text { para conseguir financier el juego } 0 \\
\text { pagar deudas de juego? }\end{array}$ & 0,0 & 0,0 & 0,0 & 82,6 & 17,3 \\
\hline $\begin{array}{l}\text { ¿Se ha arriesgado a perder relaciones } \\
\text { importantes, trabajo u oportunidaes de } \\
\text { trabajo o estudio a causa del juego? }\end{array}$ & 0,0 & 0,0 & 0,0 & 82,6 & 17,3 \\
\hline $\begin{array}{l}\text { ¿Ha pedido dinero a otros para } \\
\text { ayudarle con una situación financiera } \\
\text { desesperada provocada por el juego? }\end{array}$ & 0,0 & 0,0 & 0,1 & 82,5 & 17,3 \\
\hline
\end{tabular}

Fuente: Gómez Yáñez, J. A., Cases, J. I., Gusano, G., Lalanda, C. (2015 y 2016)

Los resultados de la tabulación dicotómica que se recogían en la Tabla 3, queda a medio camino entre el sistema DSM-IV Continuous Scoring y el
PGSI, tanto en 2015 como en 2016. El contraste de esta tabulación con el PGSI es muy consistente $\left(X^{2}=135,43 ; \varphi^{2}=1,64 ; C=0,79\right)$ (Tabla 9). 
Tabla 9. Consistencia entre los niveles de riesgo detectados según los cuestionarios PGSI Y DSM-IV (tabulación dicotómica) (\%) (n=2014)

\begin{tabular}{l|l|r|r|r|r|r|r}
\hline & & \multicolumn{7}{|c}{ Nivel de riesgo PGSI } \\
\hline & No jugador & $\begin{array}{c}\text { Sin } \\
\text { problema }\end{array}$ & Bajo riesgo & $\begin{array}{c}\text { Riesgo } \\
\text { moderado }\end{array}$ & Problemático & $\begin{array}{c}\text { Total DSM-IV } \\
\text { Dicotómico }\end{array}$ \\
\hline & No jugador & 17,3 & 0,0 & 0,0 & 0,0 & 0,0 & 17,3 \\
\cline { 2 - 9 } & Sin problema & 0,0 & 78,4 & 2,2 & 0,3 & 0,0 & 80,9 \\
\cline { 2 - 9 } $\begin{array}{c}\text { Nivel riesg0 } \\
\text { DSM-IV } \\
\text { Dicotómica }\end{array}$ & Riesgo moderado & 0,0 & 1,1 & 0,3 & 0,2 & 0,0 & 1,6 \\
\cline { 2 - 9 } & Problemático & 0,0 & 0,0 & 0,0 & 0,0 & 0,1 & 0,1 \\
\hline TOTAL PGSI & 17,3 & 79,5 & 2,5 & 0,5 & 0,2 & 100 \\
\hline
\end{tabular}

Fuente: Gómez Yáñez, J. A., Cases, J. I., Gusano, G., Lalanda, C. (2015 y 2016)

Tabla 10. Puntuaciones del cuestionario DSM-IV (Continuous Scoring) En España y Gran Bretaña (\%)

\begin{tabular}{l|c|c|c}
\hline Nivel de riesgo & Puntuación & España (2015/16) & Gran Bretaña (2010) \\
\hline Sin problema & No jugadores & 17,3 & \\
\hline Bajo riesgo & 0 & 71,0 & 91,9 \\
\hline & 1 & 5,6 & 3,9 \\
\hline Riesgo moderado & 2 & 3,0 & 1,7 \\
\hline & 3 & 1,3 & 0,7 \\
\hline & 4 & 0,6 & 0,4 \\
\hline Jugador problemático & 5 & 0,3 & 0,4 \\
\hline & 6 & 0,3 & 0,2 \\
\hline & 7 & 0,3 & 0,2 \\
\hline (n) & 11 a 13 & 0,2 & 0,4 \\
\hline & 14 a 16 & 0 & 0,1 \\
\hline & 17 y más & 0,1 & 0 \\
\hline & & $(2014)$ & 0,2 \\
\hline & & & $(7747)$ \\
\hline
\end{tabular}

Fuente: España: Gómez Yáñez, J. A., Cases, J. I., Gusano, G., Lalanda, C. (2015 y 2016). Gran Bretaña: Wardle, H. et al., (2011) 
Tabla 11 . Consistencia de los niveles de riesgo detectados según los cuestionarios PGSI Y DSM-IV (Continuous Scoring) $(\%)(n=2.014)$

\begin{tabular}{c|l|c|c|c|c|c|c}
\hline & & \multicolumn{7}{|c}{ Nivel de riesgo PGSI } \\
\hline & & $\begin{array}{c}\text { No } \\
\text { jugador }\end{array}$ & $\begin{array}{c}\text { Sin } \\
\text { problema }\end{array}$ & $\begin{array}{c}\text { Bajo } \\
\text { riesgo }\end{array}$ & $\begin{array}{c}\text { Riesgo } \\
\text { moderado }\end{array}$ & Problemático & $\begin{array}{c}\text { Total DSM-IV } \\
\text { Cont. Scoring }\end{array}$ \\
\hline \multirow{4}{*}{$\begin{array}{c}\text { Nivel riesgo } \\
\text { DSM-IV } \\
\text { (Cont. } \\
\text { Scoring) }\end{array}$} & No jugador & $\mathbf{1 7 , 3}$ & 0,0 & 0,0 & 0,0 & 0,0 & 17,3 \\
\cline { 2 - 8 } & Sin problema & 0,0 & $\mathbf{6 9 , 7}$ & $\mathbf{1 , 1}$ & $\mathbf{0 , 1}$ & 0,0 & 71,0 \\
\cline { 2 - 8 } & Bajo Riesgo & 0,0 & $\mathbf{7 , 8}$ & $\mathbf{0 , 7}$ & $\mathbf{0 , 1}$ & 0,0 & 8,5 \\
\cline { 2 - 8 } & Miesgo & 0,0 & $\mathbf{2 , 0}$ & $\mathbf{0 , 6}$ & $\mathbf{0 , 2}$ & $\mathbf{0 , 1}$ & 3,0 \\
\hline & Problemático & 0,0 & 0,0 & 0,0 & 0,0 & $\mathbf{0 , 1}$ & 0,2 \\
\hline Total PGSI & & 17,3 & 79,5 & 2,5 & 0,5 & 0,2 & 100 \\
\hline
\end{tabular}

Fuente: Gómez Yáñez, J. A., Cases, J. I., Gusano, G., Lalanda, C. (2015 y 2016).

El análisis Continuous Scoring muestra una incidencia del juego problemático y del riesgo moderado algo más elevada que en la anterior y que en el PGSI. La comparación del desglose de puntuaciones entre España y Gran Bretaña muestra niveles similares (Tabla 10). En España, los jugadores problemáticos pueden estimarse en un $0,3 \%$ de la población entre 18 y 75 años (unos 90000 individuos) y en el Reino Unido en un $0,7 \%$ de los mayores de 16 años, en "riesgo moderado" en España se detecta un 3,0\%, es decir, unos 120000 individuos.

La consistencia entre el DSM-IV Continuous Scoring y el PGSI es muy alta, casi todos los entrevistados coinciden en los niveles asignados de riesgo, con pocas excepciones que se desplazan hacia el contiguo, no obstante, es algo inferior a la que se obtenía con la tabulación dicotómica ( $X^{2}=53,3$; $\varphi^{2}=0,64 ; C=0,63$ ) (Tabla 11).

El perfil sociológico de los niveles de sensibilidad al juego según esta tabulación (Tabla 12) coincide con el PGSI (Tabla 7). El juego problemático se concentra en un reducido fragmento de la población compuesto mayoritariamente por varones jóvenes menores de 25 años, 0 entre 25 y 35 . El segmento de "riesgo moderado" se prolonga hasta los 45 años. Los dos cuestionarios coinciden en que este proble- ma gravita sobre los jugadores cotidianos (que declaran jugar varias veces a la semana) 0 semanales. A diferencia del PGSI, el DSM-IV muestra cierta incidencia del juego problemático en los individuos que residen en hogares con problemas económicos, pero este dato hay que matizarlo enérgicamente ya que se refiere a un solo individuo de la muestra.

En definitiva, los dos cuestionarios llevan a conclusiones similares.

\section{Comparación internacional}

¿En qué posición se sitúa España respecto a otros países? La Tabla 13 sintetiza estudios realizados en los últimos años ${ }^{9}$. Se desprende que en España la incidencia del juego problemático es baja, al nivel de Suecia, Francia, Canadá o Nueva Zelanda, por debajo de Estados Unidos, Islandia 0 los países asiáticos.

9 Williams, Volberg y Stevens, 2012, analizaron 242 estudios realizados hasta 2012. № obstante, se han localizado varios que escaparon a estos autores (indiscutibles expertos en la materia), lo que da idea de la cantidad de material empírico disponible. Ver Ross, D., 2012, 349-350. 
Tabla 12. Perfil sociológico por niveles de riesgo según el cuestionario DSM-IV (Continuous Scoring) (2015-16) (\%)

\begin{tabular}{|c|c|c|c|c|c|c|}
\hline & Problemáticos & $\begin{array}{c}\text { Riesgo } \\
\text { moderado }\end{array}$ & $\begin{array}{l}\text { Bajo } \\
\text { riesgo }\end{array}$ & $\begin{array}{c}\text { Sin } \\
\text { problema }\end{array}$ & $\begin{array}{c}\text { No } \\
\text { jugadores }\end{array}$ & (n) \\
\hline Total & 0,2 & 3,0 & 8,5 & 71,0 & 17,3 & (2014) \\
\hline \multicolumn{7}{|l|}{ Sexo: } \\
\hline Hombres & 0,4 & 4,0 & 9,0 & 69,3 & 17,3 & (1006) \\
\hline Mujeres & 0,1 & 2,0 & 8,0 & 72,6 & 17,3 & (1008) \\
\hline \multicolumn{7}{|l|}{ Edad: } \\
\hline De 18 a 24 años & 2,9 & 3,5 & 9,4 & 48,8 & 35,3 & (170) \\
\hline De 25 a 34 años & 0,0 & 3,4 & 13,0 & 62,6 & 21,0 & (353) \\
\hline De 35 a 44 años & 0,0 & 4,4 & 6,9 & 73,4 & 15,3 & (523) \\
\hline De 45 a 54 años & 0,0 & 1,7 & 7,6 & 77,1 & 13,6 & (419) \\
\hline De 55 a 64 años & 0,0 & 2,7 & 9,7 & 75,7 & 12,0 & (259) \\
\hline De 65 a 75 años & 0,0 & 1,7 & 5,9 & 76,6 & 15,9 & (290) \\
\hline \multicolumn{7}{|l|}{ Status Social: } \\
\hline Status alto & 0,0 & 3,1 & 7,5 & 76,1 & 13,3 & (255) \\
\hline Status medio alto & 0,3 & 2,6 & 8,9 & 73,2 & 14,9 & (302) \\
\hline Status medio medio & 0,5 & 3,2 & 8,2 & 70,2 & 17,8 & (752) \\
\hline Status medio bajo & 0,0 & 3,3 & 11,5 & 66,3 & 18,9 & (338) \\
\hline Status bajo & 0,0 & 2,5 & 6,8 & 71,4 & 19,3 & (367) \\
\hline
\end{tabular}

Tipología de jugador según la frecuencia de juego declarada

\begin{tabular}{l|c|c|c|c|c|c}
\hline Cotidianos & 1,1 & 8,0 & 13,8 & 77,0 & 0,0 & (87) \\
\hline Semanales & 0,5 & 5,9 & 13,6 & 80,0 & 0,0 & (404) \\
\hline Esporádicos & 0,4 & 5,6 & 14,3 & 79,7 & 0,0 & $(251)$ \\
\hline Infrecuentes & 0,1 & 1,5 & 7,3 & 91,0 & 0,0 & (845) \\
\hline Se consideran "no jugadores" & 0,0 & 0,5 & 1,6 & 16,4 & 81,5 & $(427)$ \\
\hline \multicolumn{2}{l|}{ Personas que viven en hogares con retrasos en: } & & & & & \\
\hline -Pago de hipotecas & 2,3 & 2,3 & 4,5 & 65,9 & 25,0 & (44) \\
\hline -Pago de compras & 0,0 & 0,0 & 4,0 & 84,0 & 12,0 & (25) \\
\hline -Pago de servicios/suministros & 1,7 & 5,2 & 12,1 & 69,0 & 12,1 & (58) \\
\hline
\end{tabular}

Fuente: Gómez Yáñez, J. A., Cases, J. I., Gusano, G., Lalanda, C. (2015 y 2016) 
Tabla 13. Estudios recientes sobre incidencia del juego problemático en la población adulta

\begin{tabular}{|c|c|c|c|c|c|c|c|}
\hline Estado / Comunidad & Año & Entrevista & Muestra (n) & $\begin{array}{l}\text { (\% pobl. } \\
\text { afectada) }\end{array}$ & $\begin{array}{c}\text { Sistema } \\
\text { estimación }\end{array}$ & Población & Ámbito temporal \\
\hline Alemania & 2015 & CATI & 11501 & 0,4 & SOGS & $16-70$ & Último año. \\
\hline \multicolumn{8}{|l|}{ Argentina } \\
\hline Buenos Aires. Á. Met. & 2015 & Personal & 1000 & $0,8 / 1,2 / 1,0$ & PGSI/DSM-IV/SOGS & $>18$ & Último año. \\
\hline \multicolumn{8}{|l|}{ Australia } \\
\hline ACT (Au. Cap. Ter) & 2009 & CATI & 5500 & 0,5 & CPGI & $>18$ & Último año. \\
\hline New South Wales & 2010 & CATI & 10000 & 0,8 & PGSI & $>18$ & Último año. \\
\hline Queensland & 2012 & CATI & 15000 & 0,5 & CPGI & $>18$ & Último año. \\
\hline South Australia & 2012 & CATI & 9508 & 0,6 & PGSI & $>16$ & Último año. \\
\hline Victoria & 2012 & CATI & 12474 & 0.9 & PGSI & $>18$ & Último año. \\
\hline Brasil & 2010 & & 3007 & 1,0 & DSM-IV & & Último año. \\
\hline Canadá & 2007 & CATI & 8496 & $0,8 / 0,9 / 1,0$ & CPGI/DSM-IV/SOGS & $>18$ & Último año. \\
\hline Corea del Sur & 2011 & CATI - online & $4000-4000$ & 1,0 & CPGI & $>19$ & Último año \\
\hline \multirow[t]{4}{*}{ España } & 2016 & CATI & 1000 & $0,1 / 0,3$ & PGSI/DSM IV & $18-75$ & Último año. \\
\hline & 2015 & Mall Int. & 7121 & 0,3 & NODS & $>18$ & Último año. \\
\hline & 2015 & CATI & 1000 & $0,1 / 0,4$ & PGSI/DSM IV & $18-75$ & Último año. \\
\hline & 2013 & Mall Int. & 3000 & 1,1 & NODS & $>18$ & Toda la vida. \\
\hline Estados Unidos & 2008 & CATI & 3435 & 0,6 & DSM IV & $>18$ & Último año \\
\hline Finlandia & 2012 & CATI & 4484 & 0,6 & PGSI & $15-74$ & Último año. \\
\hline Francia & 2014 & CATI & 15636 & 0,5 & $\mathrm{CPGl}$ & $15-75$ & Último año \\
\hline Gran Bretaña & 2010 & CATI & 7756 & $0,7 / 0,4$ & PGSI/DSM IV & & Último año \\
\hline Gales & 2015 & Personal & 4048 & 1,1 & PGSI/DSM IV & $>16$ & Último año \\
\hline Irlanda del Norte & 2010 & Personal & 1032 & 2,2 & CPGI & $>16$ & \\
\hline Hong Kong & 2011 & CATI & 2024 & 1,4 & DSM IV & $15-64$ & Último año \\
\hline Hungría & 2007 & Personal. Autoadm. & 2710 & 1,4 & SOGS & $18-64$ & Toda la vida. \\
\hline Islandia & 2007 & CATI & 3009 & 1,6 & CPGI & $18-70$ & Último año \\
\hline Italia & 2012 & & 1881 & 1,6 & SOGS-PGSI & $18-64$ & Último año \\
\hline Macao & 2003 & CATI & 1121 & 4,3 & & $15-64$ & \\
\hline Noruega & 2010 & Postal & 4636 & $2,1 / 0,2$ & $\mathrm{CPGl}$ & $15-70$ & Último año \\
\hline Nueva Zelanda & 2012 & Personal & 6251 & 0,7 & PGSI & $>18$ & Último año \\
\hline Singapur & 2011 & Personal & 3315 & 1,4 & & $>18$ & Último año. \\
\hline Sudáfrica & 2008 & Personal & 3000 & 3,0 & CPGI & $>18$ & \\
\hline Suecia & 2009 & CATI-Postal & 9917 & $0,3 / 0,8$ & CPGI/SOGS & $16-84$ & Último año. \\
\hline Suiza & 2007 & & 4497 & 0,8 & NODS & $>14$ & Último año, \\
\hline
\end{tabular}

Fuentes: Ver Anexo 2. 
¿Qué explica esta baja incidencia del juego problemático en España? Una hipótesis es que los españoles están muy familiarizados con el juego, especialmente los de suerte. "En España juega prácticamente todo el mundo. Casi el $80 \%$ de la población (entre 18 y 75 años) participa en algún sorteo 0 se acerca a algún local de juego ... La relación de los españoles con el juego es muy racional, ... más de dos siglos y medio de juegos organizados por el Estado han convertido el juego en algo normal ... y les ha enseñado que, jugando, se pierde a la larga" (Gómez Yáñez et al., 2015: 9). La cultura del juego española se basa en los de suerte (loterías de Navidad, El Niño, semanales, los cupones de la ONCE y loterías primitivas) más que en los de habilidad. Son juegos en los que normalmente, se pierde, aunque se puedan conseguir grandes premios que mantienen la ilusión. Esta preeminencia de los juegos de suerte sobre los de habilidad también parece explicar las bajas tasas de juego problemático en países como Suecia. Volberg et al. (2001: 255) argumentaban que: "una posible explicación es que la gran disponibilidad de juegos basados en la suerte (loterías, ruletas en restaurant casinos) más que los basados en la ha- bilidad 0 en un mix de habilidad y suerte, ha contribuido a la relativamente baja tasa de prevalencia del juego problemático. Investigaciones en varios países sugieren que formas de juego que impliquen altos niveles de habilidad 0 una mezcla entre la suerte y la habilidad están asociados a altos riesgos de juego problemático más que los basados puramente en la suerte". Es decir, en los juegos de suerte el cliente deja que decida la fortuna, mientras que en los de habilidad cree poder controlar el juego, lo que tiende a favorecer conductas adictivas 0 competitivas y a desencadenar la pérdida de control.

\section{EL JUEGO ONLINE}

\section{Las características de los jugadores online}

Se habla del juego online como algo homogéneo pero no lo es. En Internet conviven varios mercados en los que los clientes practican sus juegos preferidos, pero no pasan de unos a otros más allá de la curiosidad (Figura 3). El juego presencial tampoco es homogéneo, cada persona juega lo que le gusta.

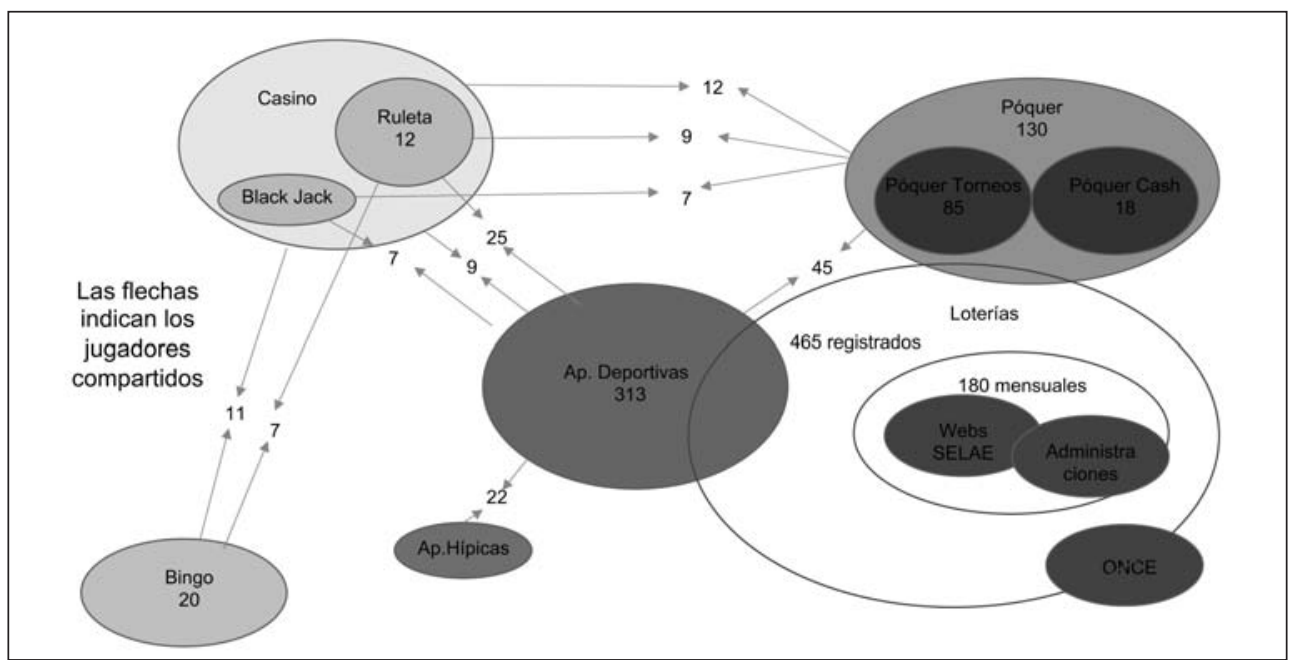

Figura 3. Jugadores online según juegos (miles)

Fuente: Elaboración propia a partir de DGOJ: Análisis del perfil del jugador online, Madrid, julio 2015. SELAE: Memoria Anual 2014. Nota: No se han incluido en el gráfico los jugadores que juegan a varios juegos que, por tanto, se puede decir que flotan sobre todos ellos. 


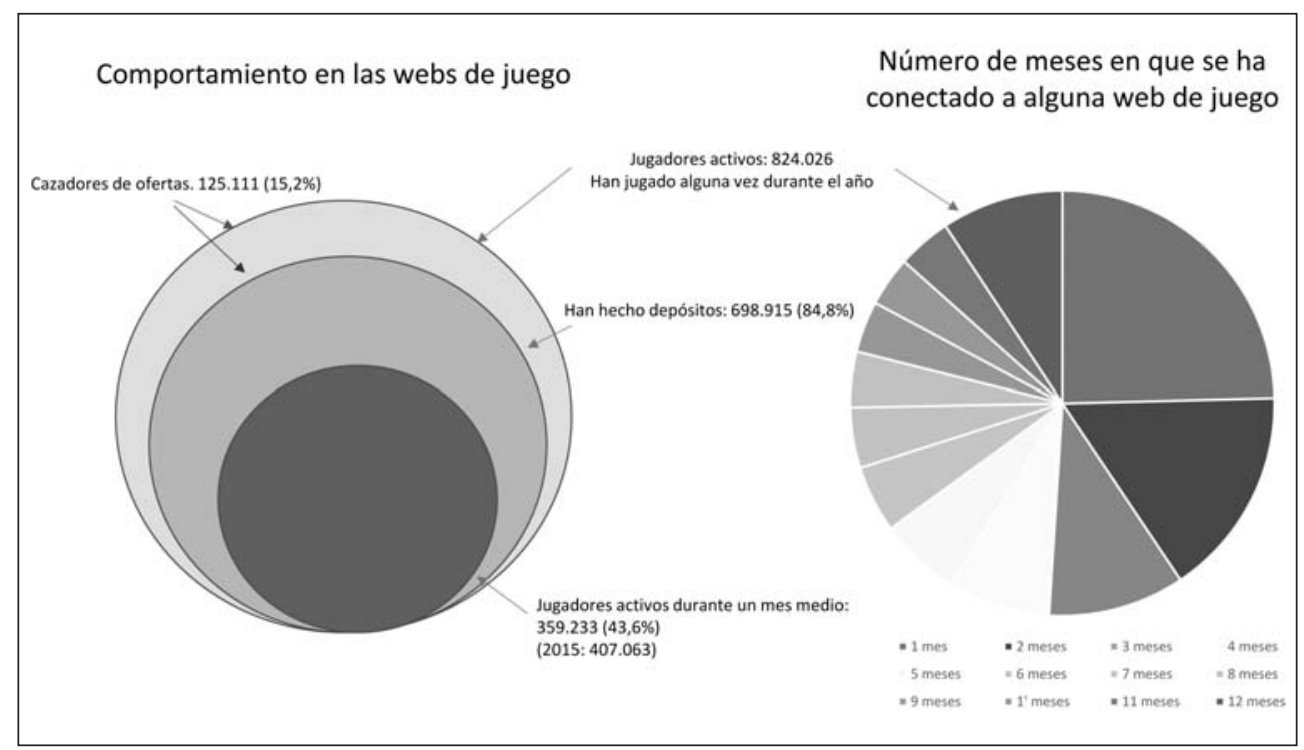

Figura 4. Jugadores online activos (2014)

Fuente: Dirección General de Ordenación del Juego

Los jugadores online componen un conjunto difuso cuyos límites no están definidos ni sus integrantes participan con la misma intensidad. Su número no es fácil de precisar. Según la Dirección General de Ordenación del Juego del Ministerio de Hacienda, a partir de la información de las webs autorizadas, en 2014 hubo 824026 jugadores activos ${ }^{10}$ (jugaron alguna vez durante el año), registrados en una media de 2,5 webs (es decir, había 2088496 registros). Más de la mitad se dio de alta durante el año (425 728) lo que da idea de elevada rotación. Arriesgaron dinero, $698915^{11}$, el 84,8\%; el 15,2\% restante se puede considerar "cazadores de ofertas", jugaron bonos de promoción de alguna web, perdieron y no volvieron a jugar. Un mes medio jugaron el 43,6\%

10 Dirección General de Ordenación del Juego (2015): Análisis del perfil del jugador online, Ministerio de Hacienda y Administraciones Públicas, Madrid, 19.

11 DGOJ, Ibídem, pág. 45. de los jugadores activos (359 283 personas ${ }^{12}$ ). La frecuencia de juego es baja, el 51,0\% jugó en alguna ocasión en tres meses 0 menos durante el año, lo que indica que son jugadores online light, casi curiosos. En el otro extremo, jugaron más de diez meses 141885 individuos, el 17,2\% de los inscritos ${ }^{13}$. A estos se les podría considerar heavy users de juego online y, por ende, susceptibles de superar los umbrales de juego problemático 0 riesgo moderado. En definitiva, entre quienes se conectan a las webs de juego hay grandes diferencias en patrones de juego y, lógicamente, de niveles de riesgo (Figura 4).

Los datos de 2015 muestran que el número de jugadores activos un mes medio ascendió a 407 063, es decir, creció respecto al año anterior un $13,3 \%$, básicamente debido a la atracción de las apuestas deportivas ${ }^{14}$.

12 DGOJ (2015). Análisis global del mercado nacional de juego online, abril-junio 2015, Madrid, 29.

13 Ibídem, Fé de erratas que modificaba la Tabla 2.

14 DGOJ (2015). Análisis global, pág. 29. 
Es un colectivo muy masculino $(87,0 \%)$ y joven, se divide en tercios algo desiguales: menores de 25 años $(29,2 \%)$, de 26 a $35(39,4 \%)$ y mayores de 35 $(31,4 \%)$. La edad media de las mujeres es mayor que la de los hombres: 34,7 años frente a 32,015. Los jugadores online activos representan el 3,9\% de los hombres y el 0,5\% de las mujeres mayores de 18 años. Entre los hombres menores de 35 años suman en torno $10 \%$.

\section{Metodología}

En las ediciones de 2015 y 2016 del estudio Percepción social sobre el juego de azar en España se incluyeron dos muestras para analizar el colectivo de jugadores online, de 469 y 616 individuos respectivamente $(n=1085)$. La segunda reproducía fielmente la estructura por edad y sexo del universo de jugadores online, la primera tenía algunos sesgos derivados de la ausencia de datos de referencia y, por tanto, se tomó como referencia el perfil de los jugadores online que se deducía de la muestra a la población entre 18 y 75 años ya comentada. Los trabajos de campo consistieron en una encuesta online auto-administrada a la que podían acceder los individuos que hubieran jugado arriesgando dinero en los dos meses anteriores. Se aplicaron los cuestionarios PGSI y DSM-IV.

\section{El juego problemático online en España}

A partir de las encuestas se deduce que quienes acceden a webs de juego tienen un fuerte sesgo hacia los estratos sociales medio alto y alto (hogares cuyo sustentador principal tiene empleo estable, con niveles de estudios altos y elevadas cualificaciones profesionales ya sean por cuenta propia 0 ajena) (Tabla 14). En este perfil coinciden todos los estudios sobre el juego online Petry et al., 2007; Griffiths et al., 2009; Lloyd et al., 2010; Hing et al., 2014).

Tabla 14. Status y origen de los jugadores online (\%)

\begin{tabular}{|c|c|c|c|c|c|}
\hline & 2011 & 2012 & 2013 & 2014 & 2015 \\
\hline Status alto & 15,9 & 21,0 & 18,8 & 16,8 & 13,3 \\
\hline Status medio alto & 40,0 & 49,5 & 43,2 & 49,3 & 38,7 \\
\hline Status medio medio & 27,4 & 22,1 & 23,7 & 25,8 & 22,4 \\
\hline Status medio bajo & 9,3 & 7,2 & 6,8 & 5,1 & 8,6 \\
\hline Status bajo & 0,4 & 0,2 & 0,6 & 0,4 & 0,6 \\
\hline Sin información & 7,0 & & 6,9 & 2,6 & 16,4 \\
\hline (n) & $(460)$ & (472) & (468) & (469) & (616) \\
\hline
\end{tabular}

Fuente: Gómez Yáñez, J. A., Cases, J. I., Gusano, G., Lalanda, C. (2015 y 2016) $)^{15}$

15 DG0J (2015). Análisis del perfil, pág. 25. 
Están en zona de juego problemático entre el $6,1 \%$ y el $16,1 \%$ de los jugadores online, aplicando Ios cuestionarios PGSI o DSM-IV (explotación dicotómica). Los dos criterios de clasificación coinci- den en mostrar alrededor de un $20 \%$ en niveles de riesgo moderado en el juego online. La consistencia entre los dos criterios de clasificación es elevada: ( $X^{2}=64,99 ; \varphi^{2}=0,65 ; C=0,63$ ) (Tabla 15).

Tabla 15. Consistencia entre los niveles de riesgo en juego online detectados según los cuestionarios PGSI y DSM-IV (tabulación dicotómica) (\%) $(\mathrm{n}=1085)$

\begin{tabular}{c|l|c|c|c|c|c}
\hline \multirow{2}{*}{} & \multicolumn{4}{c|}{ Nivel de riesgo según PGSI } & \multirow{2}{*}{ Total DSM-IV } \\
\cline { 3 - 7 } & & Sin problema & Bajo riesgo & $\begin{array}{c}\text { Riesgo } \\
\text { moderado }\end{array}$ & Problemático & Dicotómico \\
\hline \multirow{2}{*}{$\begin{array}{c}\text { Nivel riesgo } \\
\text { DSM-IV } \\
\text { Dicotómica }\end{array}$} & Sin problema & 28,1 & 27,3 & 13,7 & 5,5 & 74,6 \\
\cline { 2 - 7 } & Riesgo moderado & 2,7 & 4,9 & 6,2 & 5,4 & 19,2 \\
\cline { 2 - 7 } & Problemático & 0,0 & 0,1 & 0,8 & 5,3 & 6,2 \\
\hline TOTAL PGSI & 30,8 & 32,3 & 20,8 & 16,1 & 100 \\
\hline
\end{tabular}

Fuente: Gómez Yáñez, J. A., Cases, J. I., Gusano, G., Lalanda, C. (2015 y 2016)

Tabla 16. Comparación de la incidencia del juego problemático online en España, Canadá y Australia (\%)

\begin{tabular}{l|c|c|c|c|c|c}
\hline & \multicolumn{2}{|c|}{ España } & Canadá & \multicolumn{3}{c}{ Australia } \\
\hline Cuestionario & PGSI & $\begin{array}{c}\text { DSM-IV } \\
\text { (Dicotómico) }\end{array}$ & CPGI & \multicolumn{2}{c}{ PGSI } \\
\hline & $2015-16$ & $2015-16$ & $2006-07$ & $\begin{array}{c}2015 \\
\text { (online) }\end{array}$ & $\begin{array}{c}2015 \\
\text { (mixtos) }\end{array}$ & $\begin{array}{c}2014 \\
\text { (Interactive) }\end{array}$ \\
\hline Sin problema & 30,8 & 74,6 & 45,0 & 46,1 & 32,1 & 58,0 \\
\hline Jugador de bajo riesgo & 32,3 & & 37,9 & 23,7 & 25,5 & 25,0 \\
\hline Jugador de riesgo moderado & 20,8 & 19,2 & 12,6 & 22,0 & 26,7 & 14,0 \\
\hline Jugador problemático & 16,1 & 6,2 & 4,5 & 8,2 & 15,7 & 3,0 \\
\hline (n) & $(1085)$ & $(1085)$ & $(179)$ & $(608)$ & $(2570)$ & \\
\hline
\end{tabular}

Fuentes: España: Gómez Yáñez, J. A., Cases, J. I., Gusano, G., Lalanda, C. (2015 y 2016). Canadá: Wood, R. T., Williams, R. J. (2009). Australia: Blaszczynski, A. et al. (2015) y Gainsbury, S. (2014) 
Estas tasas de juego problemático son similares a las halladas en otros países (Tabla 16). № hay diferencia significativa con Australia (Blaszczynski et al., 2015), aplicando el cuestionario PGSI al compararlas con el resultado de un estudio sobre jugadores mixtos (juegan online y en presencial), porque, en realidad, los jugadores online españoles son mixtos. En aquel país, sin embargo, en el mismo año, se encontró una tasa más baja entre los jugadores por todos los medios interactivos (móviles, Iphone, Ipad, PC) (Gainsbury, 2015) (3,0\%). En Canadá, en 2006, se hallaron unas tasas más bajas (4,5\%) (Wood y Williams, 2009). En Italia, en 2009, Carboni (2012) estimó un 4,9\% de juego problemático online. En Noruega, un 6,5\% en 2006 (Sandven, 2007). Se podría llegar a dos conclusiones. La primera, que las tasas de juego problemático online son más elevadas que en el juego presencial, algo observado desde hace tiempo (Wood y Williams, 2009: 83, 90-91). La segunda, que los datos sobre España no sobrepasan los de otros países, sobre todo si se descartan los sesgos metodológicos inherentes a estas investigaciones.

Las comparaciones internacionales deben tomarse con precaución, el relativo caos metodológico del que se habló más arriba se ha trasladado a las investigaciones sobre el juego online ya que los sistemas de muestreo factibles en los trabajos de campo han sido diferentes por la dificultad de ajustarlos a los datos reales.

Las Tablas 17 y 18 recogen las respuestas a los dos cuestionarios.

Tabla 17. Respuestas a los items del cuestionario PGSI de los jugadores online (2015-16) (\%) ( $n=1.085)$

\begin{tabular}{l|c|c|c|c}
\hline ¿En los últimos doce meses ...? & Casi siempre & Con frecuencia & Alguna vez & Nunca \\
\hline $\begin{array}{l}\text { ¿Ha vuelto para intentar ganar el dinero que } \\
\text { perdió? }\end{array}$ & 4,9 & 11,4 & 43,3 & 40,4 \\
\hline $\begin{array}{l}\text { ¿Se ha sentido culpable sobre la forma en que } \\
\text { juega y lo que sucede cuando juega? }\end{array}$ & 1,8 & 6,8 & 21,5 & 69,9 \\
\hline $\begin{array}{l}\text { ¿Ha habido gente que le ha criticado lo que } \\
\text { juega, o le ha dicho que tiene un problema con } \\
\text { el juego, con independencia de que usted piense } \\
\text { que es verdad o no? }\end{array}$ & 2,8 & 5,2 & 20,4 & 71,6 \\
\hline $\begin{array}{l}\text { ¿Ha pensado que podía tener un problema con } \\
\text { el juego? }\end{array}$ & 2,9 & 4,6 & 18,3 & 74,2 \\
\hline $\begin{array}{l}\text { ¿Ha necesitado jugar más dinero para conseguir } \\
\text { la misma emoción? }\end{array}$ & 2,1 & 5,7 & 20,2 & 72,0 \\
\hline $\begin{array}{l}\text { ¿Ha sentido que el juego le ha producido } \\
\text { problemas de salud, incluyendo estrés 0 } \\
\text { ansiedad? }\end{array}$ & 2,4 & 4,8 & 17,0 & 75,8 \\
\hline $\begin{array}{l}\text { iHa jugado o apostado más dinero del que se } \\
\text { podía permitir perder? }\end{array}$ & 4,0 & 5,5 & 14,8 & 75,7 \\
\hline $\begin{array}{l}\text { ¿Ha tomado dinero prestado o vendido algo para } \\
\text { conseguir dinero para jugar? }\end{array}$ & 2,5 & 4,3 & 11,5 & 81,7 \\
\hline $\begin{array}{l}\text { ¿Ha sentido que el juego le ha provocado } \\
\text { problemas económicos a usted 0 a su familia? }\end{array}$ & 1,8 & 4,0 & 11,2 & 83,0 \\
\hline
\end{tabular}

Fuente: Gómez Yáñez, J. A., Cases, J. I., Gusano, G., Lalanda, C. (2015 y 2016) 
Tabla 18. Respuestas a los items del cuestionario DSM IV de los jugadores online (2015-16) (\%) ( $n=1085)$

\begin{tabular}{|c|c|c|c|c|}
\hline ¿En los últimos doce meses ...? & $\begin{array}{l}\text { Cada vez que } \\
\quad \text { pierdo }\end{array}$ & $\begin{array}{c}\text { La mayor parte } \\
\text { de las veces que } \\
\text { pierdo }\end{array}$ & $\begin{array}{c}\text { Alguna vez, } \\
\text { más o menos } \\
\text { la mitad de las } \\
\text { veces que pierdo }\end{array}$ & Nunca \\
\hline \multirow[t]{2}{*}{$\begin{array}{l}\text { Cuando usted juega, ¿con que frecuencia vuelve } \\
\text { a jugar para ganar lo que perdió otro día? }\end{array}$} & 6,3 & 23,7 & 44,7 & 25,3 \\
\hline & Con frecuencia & Algunas veces & Ocasionalmente & Nunca \\
\hline $\begin{array}{l}\text { ¿Con qué frecuencia se ha encontrado usted } \\
\text { mismo pensado sobre juego, es decir, volviendo } \\
\text { sobre partidas anteriores, planificando la } \\
\text { próxima vez que jugará o pensando formas } \\
\text { para tener dinero para jugar? }\end{array}$ & 11,1 & 35,4 & 31,3 & 22,2 \\
\hline $\begin{array}{l}\text { ¿Tiene necesidad de jugar más y más dinero } \\
\text { para conseguir la emoción que desea conseguir } \\
\text { jugando? }\end{array}$ & 3,6 & 22,5 & 23,3 & 50,6 \\
\hline $\begin{array}{l}\text { ¿Se siente usted irritable cuando intenta dejar } \\
\text { de jugar? }\end{array}$ & 4,9 & 14,0 & 17,1 & 64,0 \\
\hline $\begin{array}{l}\text { ¿Ha jugado para escapar de sus problemas } 0 \\
\text { cuando usted está deprimido, ansioso o mal } \\
\text { consigo mismo? }\end{array}$ & 5,2 & 15,4 & 16,2 & 63,2 \\
\hline $\begin{array}{l}\text { ¿Ha mentido a su familia } 0 \text { a otros para } \\
\text { esconder el tiempo que dedica a jugar? }\end{array}$ & 4,1 & 11,5 & 13,2 & 71,2 \\
\hline $\begin{array}{l}\text { ¿Ha intentado controlar, recortar lo que juega } 0 \\
\text { dejar de jugar? }\end{array}$ & 10,0 & 18,0 & 20,6 & 51,4 \\
\hline $\begin{array}{l}\text { ¿Ha cometido una falta } 0 \text { un delito para para } \\
\text { conseguir financiar el juego o pagar deudas } \\
\text { de juego? }\end{array}$ & 1,8 & 6,4 & 5,8 & 86,1 \\
\hline $\begin{array}{l}\text { ¿Se ha arriesgado a perder relaciones } \\
\text { importantes, trabajo u oportunidades de } \\
\text { trabajo o estudio a causa del juego? }\end{array}$ & 2,0 & 7,3 & 7,7 & 82,9 \\
\hline $\begin{array}{l}\text { ¿Ha pedido dinero a otros para ayudarle } \\
\text { con una situación financiera desesperada } \\
\text { provocada por el juego? }\end{array}$ & 2,5 & 6,3 & 6,6 & 84,6 \\
\hline
\end{tabular}

Fuente: Gómez Yáñez, J. A., Cases, J. I., Gusano, G., Lalanda, C. (2015 Y 2016)

Las Tablas 19 y 20 muestran que la incidencia del juego problemático es más homogénea entre los jugadores online que en los presenciales 0 entre la población en general. Ambos criterios muestran que el juego problemático afecta sobre todo a los jugadores más frecuentes, los que declaran jugar online todos los días o varias veces a la semana, desciende en paralelo al status social del entrevistado, 0 sea, a sus ingresos; y afecta casi de manera indiferenciada a ambos sexos, y más que la media a los menores de 25 años. 
Tabla 19. Perfil sociológico por niveles de riesgo de los jugadores online según el cuestionario PGSI (2015-16) (\%) $(n=1085)$

\begin{tabular}{l|c|c|c|c}
\hline & $\begin{array}{c}\text { Jugadores } \\
\text { problemáticos }\end{array}$ & Riesgo moderado & Bajo riesgo & Sin problema \\
\hline Total & 16,1 & 20,8 & 32,3 & 30,8 \\
\hline
\end{tabular}

Sexo

\begin{tabular}{l|c|c|c|c}
\hline Hombres & 16,1 & 20,5 & 32,9 & 30,5 \\
\hline Mujeres & 15,5 & 22,0 & 31,1 & 31,4 \\
\hline
\end{tabular}

Edad

\begin{tabular}{l|c|c|c|c}
\hline De 16 a 24 años & 15,3 & 27,4 & 38,7 & 18,6 \\
\hline De 25 a 34 años & 18,4 & 19,5 & 31,2 & 30,8 \\
\hline De 35 a 44 años & 17,3 & 17,3 & 28,8 & 36,5 \\
\hline 45 y más & 11,1 & 19,2 & 35,5 & 34,3 \\
\hline
\end{tabular}

Status

\begin{tabular}{l|c|c|c|c}
\hline Status alto & 16,8 & 21,8 & 28,1 & 33,3 \\
\hline Status medio alto & 15,9 & 20,6 & 31,8 & 31,7 \\
\hline Status medio medio & 14,1 & 19,3 & 34,6 & 32,1 \\
\hline Status medio bajo & 14,9 & 26,5 & 38,2 & 20,4 \\
\hline
\end{tabular}

Tipología de jugador según la frecuencia de juego que declara

\begin{tabular}{l|c|c|c|c}
\hline Cotidianos & 26,6 & 27,6 & 27,0 & 18,7 \\
\hline Semanales & 17,3 & 17,1 & 33,9 & 31,6 \\
\hline Esporádicos & 10,5 & 18,3 & 36,5 & 34,7 \\
\hline Infrecuentes & 2,3 & 17,6 & 33,2 & 46,9 \\
\hline
\end{tabular}

Fuente: Gómez Yáñez, J. A., Cases, J. I., Gusano, G., Lalanda, C. (2015 y 2016) 
Tabla 20. Perfil sociológico por niveles de riesgo de los jugadores online según el cuestionario DSM-IV (Dicotómico) $(2015-16)(\%)(n=1085)$

\begin{tabular}{|c|c|c|c|}
\hline & Jugadores problemáticos & Riesgo moderado & Sin problema \\
\hline Total & 6,2 & 19,1 & 74,7 \\
\hline \multicolumn{4}{|l|}{ Sexo } \\
\hline Hombres & 5,8 & 19,6 & 74,6 \\
\hline Mujeres & 7,5 & 16,7 & 75,9 \\
\hline \multicolumn{4}{|l|}{ Edad } \\
\hline De 16 a 24 años & 9,3 & 22,2 & 68,5 \\
\hline De 25 a 34 años & 6,9 & 20,1 & 73,0 \\
\hline De 35 a 44 años & 4,4 & 18,0 & 77,6 \\
\hline 45 y más & 5,1 & 13,1 & 81,8 \\
\hline \multicolumn{4}{|l|}{ Status } \\
\hline Status alto & 8,9 & 18,2 & 72,8 \\
\hline Status medio alto & 5,9 & 16,2 & 77,9 \\
\hline Status medio medio & 4,4 & 22,3 & 73,3 \\
\hline Status medio bajo & 3,5 & 18,1 & 78,4 \\
\hline \multicolumn{4}{|c|}{ Tipología de jugador según la frecuencia de juego que declara } \\
\hline Cotidianos & 11,9 & 23,9 & 64,2 \\
\hline Semanales & 6,8 & 16,4 & 76,8 \\
\hline Esporádicos & 1,7 & 18,3 & 80,0 \\
\hline Infrecuentes & 0,0 & 14,0 & 86,0 \\
\hline
\end{tabular}

Fuente: Gómez Yáñez, J. A., Cases, J. I., Gusano, G., Lalanda, C. (2015 y 2016) 
Elevando estos datos al conjunto de la población residente en España, se estima que se encuentran en niveles de juego problemático derivados del juego online entre el $0,1 \%$ y el $0,3 \%$, de la población comprendida entre 18 y 45 años (en términos prácticos, ya que el juego online por encima de 45 años es casi irrelevante) lo que viene a coincidir con las estimaciones para la población general en general. Se puede concluir, por tanto, que en este momento, uno de los elementos que más inciden en el juego problemático es el juego online.

\section{CONCLUSIONES}

Desde 1990 el juego problemático en España se ha reducido desde aproximadamente el 2,0\% de la población adulta al 0,3\% en 2015-16, una tasa baja en comparación internacional. Ha cambiado el perfil de los afectados, de individuos de clases medias y medias bajas de generaciones intermedias a menores de 35 años. Los juegos sobre los que gravita el problema han pasado de las máquinas tragamonedas al juego online. Las tasas de juego problemático online están al nivel de las de Australia, Canadá 0 Italia (países sobre los que hay datos).

La sociología del juego problemático avanza de estudio empírico a estudio empírico (encuesta a encuesta). Este trabajo es el primero que muestra los 20 estudios sobre el tema que se han hecho en España desde 1990. Lo habitual en la literatura es citar ocho o diez que, por reiteración, se han convertido en referentes, que muestran tasas de juego problemático más elevadas que los marginados de la literatura académica. Se podría hablar de un sesgo selectivo para presentar el problema con mayor dimensión de la real.

Precisar la población realmente afectada tiene implicaciones. Es un tema con elevada carga emocional perceptible en algunos círculos académicos, en las administraciones públicas y en las asociaciones de afectados. Este enfoque lleva a que las administraciones tomen medidas teóricamente preventivas destinadas a públicos amplísimos a los que se califica de vulnerables (jóvenes, mujeres, etc.) que suelen ser los colectivos sociales que menos juegan, lo que da idea del desenfoque.
Esto conduce a políticas caras e ineficaces. En todo caso, la evolución del problema muestra que las Administraciones y las empresas de juego gestionan muy correctamente el tema.

El juego problemático no está asociado a grandes sectores sociales. Como se ha descrito, alcanza a entre 30000 y 100000 personas. Los datos sobre la población atendida sitúan la cifra entre los 15000 y los 20000 .

Algunos políticos y responsables de administraciones públicas relacionadas con el juego consideran que su función en la materia es proteger a los ludópatas. Para ello, suelen basarse en escritos académicos que reiteran estudios desfasados, que exageran la dimensión cuantitativa del problema. La prevención del juego problemático requiere políticas públicas "de precisión" en las que la colaboración con las empresas públicas y privadas que gestionan el juego es esencial.

\section{AGRADECIMIENTOS}

Debo agradecer al profesor don José Ignacio Cases la oportunidad que me ofreció para investigar sobre el juego. Sin su confianza y conocimiento del tema este trabajo y varias investigaciones no hubieran sido posibles. La colaboración con don Germán Gusano Serrano, director de la Fundación Codere, ha sido siempre especialmente productiva por su conocimiento sobre la responsabilidad corporativa en esta industria. De don Carlos Lalanda me sorprende su conocimiento de los recovecos de la industria del juego. De los tres hay ideas en este trabajo, sin que logre separarlas de las mías. Tengo el placer de formar parte, con ellos, del grupo de trabajo sobre juego del Instituto de Política y Gobernanza de la Universidad Carlos III, que ha dado lugar a numerosos estudios. Las investigaciones que sirven de base a la parte empírica de este trabajo fueron financiadas por la Fundación Codere.

\section{REFERENCIAS BIBLIOGRÁFICAS}

Abbott, M. W. (2006). Do EGMs and problem gambling go together like a horse and carriage? Gambling Research, 18 (1), 7-38. 
Abbott, M. W. y Volberg, R. A. (1996). The New Zealand National Survey of problem and pathological gambling. Journal of Gambling Studies, 12, 143-160.

Abbott, M. W., Williams, M., y Volberg, R. A. (2004). A prospective study of problem and regular no problem gamblers living in the community. Substance use and measure, 39 (6), 855-884.

Append (2001). Estudio sobre la prevalencia de la adicción al juego en Álava, Vitoria: ASAJER.

Arbinaga, F. (2000). Consumo de drogas y juego patológico en Punta Umbría (Huelva). Revista Española de Drogodependencias, 25 (3), 301-320.

Barroso, C. (2003). Las bases sociales de la ludopatía, Granada: Universidad de Granada.

Becoña, E. (2004a). Prevalencia del juego patológico en Galicia mediante NODS. ¿Descenso de la prevalencia 0 mejor evaluación del trastorno? Adicciones, 6, 3, 173-184.

Becoña, E. (2004b). El juego patológico: prevalencia en España. Salud y drogas, 4, 2, 9-34.

Becoña, E. (1993). El juego compulsivo en la comunidad autónoma gallega, Santiago de Compostela, Consellería de Sanidade da Xunta de Galicia. Versión en inglés: (1993). The prevalence of pathological gambling in Galicia. Journal of Gambling Studies, 9, 353-369.

Becoña, E. y Fuentes, M. J. (1995). El juego patológico en Galicia evaluado con el South Oaks Gambling Screen. Adicciones, 7, 423-440.

Black, D. W., Shaw, M., y Blum, N. (2010). Pathological gambling and compulsive buying: do they fall within an obsessive-compulsive spectrum. Dialogues Clin. Neuroscience, 2010, 12 (2), 175-185.

Blanco, C., Moreyra, P., Nunes, E. V., Sáiz-Ruiz, J. e Ibáñez, A. (2001). Pathological gambling, addiction or compulsión? Semin. Clin. Neuropsychiatry, Jul. 6 (3), 167-76.

Blaszczynski, A., Russell, A., Gainsbury, S. y Hing, N. (2015). Mental health and online, land based and mixed gamblers, Southern Cross University.

Bondolfi, G., Osiek, C. y Ferrero, F. (2008). Prevalence estimates of pathological gambling in Switzerland, Acta Psychiatrica Scandinavica, 117, 236-239.
Braid, K. y Volberg, R. A. (2008). British Columbia. Problem Gambling Prevalence Study, Ministry of Public Safety and Solicitor General, Ipsos Reid Public Affairs.

Carboni, G. (2012). Prevalence rate measures in remote problematic gambling. Lex Giochi, $19 / 10 / 2012$.

Cayuela, R. (1990). Characteristics and situation of gambling addiction in Spain: epidemiological and clinical aspects. VIII International Conference of Risk and Gambling, London.

Cayuela, R. y Guirado, J. L. (1991). Characteristics and situation of gambling addiction in Spain. En Eadington, W. R., Cornelius, J. A. (eds.): Gambling and public policy: International Perspectives, Reno (Nevada): University of Nevada.

Costes, J. M., Pousett, M., Eroukmanoff, V., Le Nezet, 0., Richard, J-B., Guignard, R., Beck, F. y Arwidson, P. (2011). Les niveaux et pratiques des jeux de has arc et d'argent en 2010, French Monitoring Centre for Drugs and Drugs Addiction and the National Institute for Prevention and Health Education.

Collins, P. y Barr, G. (2003). Gambling and problem gambling in South Africa: a national study, National Centre for the Study of Gambling at the University of Cape Town.

Culleton, R. P. (1989). The prevalence rates of pathological gambling: a look at methods. Journal of Gambling Behavior, 5, 22-41.

Custer, R. L. (1989). Foreword. En Shafer, H. J., Stein, S., Gambino, B. y Cummings, T. (eds.). Compulsive gambling. Theory, research and practice, 9-10, Lexington, MA, Lexington Books.

Dickerson, M. (1987). The future of gambling research, learning from the lessons of alcoholism. Journal of Gambling Behavior, 3, 248-256.

Domínguez Álvarez, A. M. (2007). Epidemiología. En Guía clínica: actuar ante el juego patológico, Sevilla: Consejería para la Igualdad y el Bienestar Social, Junta de Andalucía.

Domínguez Álvarez, A. M. (2009). Epidemiología y factores implicados en el juego patológico. Apuntes de Psicología, 27, 1, 3-20.

Echeburúa, E. (2016). Juego patológico y juego controlado (responsable). En Echeburúa, E. (coord.). Abuso de Internet. Madrid: Pirámide, 252-268. 
Echeburúa, E. (1999). ¿Adicciones sin drogas?. Bilbao: Desclée de Brouwer.

Echeburúa, E., Báez, C., Fernández, J. y Páez, D. (1994). Cuestionario de juego patológico de South Oaks (SOGS), Validación española. Análisis y Modificación de Conducta, 20, 769-791.

Eisen, S. A., Lin, N., Scherrer, J. F., Griffith, K., True, W. R., Goldberg, J. y Tsuang, M. T. (1998). Familiar influences on gambling behavior: an analysis of 3359 twin pairs. Addiction, 93 (9), 1375-84.

FEJAR (Federación Española de Jugadores de Azar Rehabilitados) (2016): Memoria de actividades 2015, Madrid.

Ferrández de la Cruz, A. (2010). Eficacia de un tratamiento psicológico multicomponente para jugadores patológicos de máquinas recreativas con premio, Madrid: Universidad Complutense de Madrid.

Ferris, J., Wynne, H. (2001). The Canadian problem Gambling Index, Final Report.

Gainsbury, S. (2014). Assessing the impact of interactive gambling and new technologies. $10^{\text {th }}$ European Conference on Gambling Studies and Policy Issues, Helsinki (Finland).

Gernstein, D., Murphy, S., Tace, M., Hoffman, J., Palmer, A., Johnson, R. et al. (1999). Gambling impact and behavior study, Chicago (II): report to the National Gambling Impact Study Commission.

Gómez Yáñez, J. A., Cases Méndez, J. I., Gusano Serrano, G. y Lalanda, C. (2016). Percepción social del juego de azar en España VII, Madrid: Instituto de Política y Gobernanza de la Universidad Carlos III.

Gómez Yáñez, J. A., Cases Méndez, J. I., Gusano Serrano, G. y Lalanda, C. (2015). Percepción social del juego de azar en España VI, Madrid: Instituto de Política y Gobernanza de la Universidad Carlos III.

Gonnerman, M. E. y Lutz, G. M. (2011). Gambling attitudes and behaviors: a 2011 survey of adults lowans, Center for social and behavioural research, Cedar Falls: University of Northern lowa.

González Ibáñez, Á. y Volberg, R. A. (2010). Estudi epidemiològic de prevalença del joc patològic a la poblaciò adulta de Catalunya (2007-2008), Barcelona, Departament de Salut de la Generalitat de Catalunya.

Gossop, M. (1989). Relapse and addictive behaviour, Londres: Routledge.

Griffiths, M. D., Wardle, H., Orford, J., Sproston, K. y Erens, B. (2009). Sociodemographic correlates of Internet gambling. Findings from the 2007 British Gambling Prevalence survey. Cyber Psychology, Behavior, and Social Networking, 12, 199-202.

Hing, N., Gainsbury, S., Blaszeynski, A., Wood, R., Lubman, D. y Russell, A. (2014). Interactive gambling, Melbourne: Gambling research Australia.

Hodgins, D. C. y El-Guebaly, N. (2000). Natural and treatment-assisted recovery from gambling problems: a comparison of resolved and active gamblers. Addiction, 95, 777-789.

Irurita, I. M. (1996). Estudio sobre la prevalencia de los jugadores de azar en Andalucía, SeviIla: Comisionado para la Droga, Consejería de Asuntos Sociales, Junta de Andalucía.

Jiménez Murcia, S., Fernández Aranda, F., Santamaría, J., Granero, R., Penelo, E., Gómez Peña, M., Aymamí, N., Moragas, L., Soto, A. y Menchón, J. M. (2012). XXV Jornada de Terapia del Comportamiento y Medicina Conductual en la práctica clínica, Barcelona.

Laans00, N. (2009). Estonia. En Meyer, G., Hayer, T. y Griffiths, M. (eds.). Problem gambling in Europe: challenges, prevention and interventions, New York: Springer.

Labrador, F. J., Becoña, E., Crespo, M., Echeburúa, E. y Labrador, M. (2016). Estudio sobre prevalencia, comportamiento y características de los usuarios de juegos de azar en España 2015, Madrid, Dirección General de Ordenación del Juego.

Labrador, F. J., Becoña, E., Crespo, M., Echeburúa, E. y Labrador, M. (2013). El juego en España 2013, Madrid: SELAE (power point).

Labrador, F. J., Bernaldo de Quirós, M., García Fernández, M., Fernández Arias, I., Estupia, F. y Labrador, M. (2016). Sistemas de evaluación y detección de problemas de juego, en Echeburúa, E. (coord.): Abuso de Internet, Madrid: Pirámide, 270-298. 
Legarda, J. J., Babiola, R. y Abreu, J. M. (1992). Prevalence estimates of pathological gambling in Seville, British Journal of Addictions, 87, 767-770.

LaPlante, D. A. y Shaffer, H. J. (2007). Understanding the influence of gambling opportunities. Expanding exposure models to include adaptation, American Journal of Orthopsychiatry, 77 (4), 616-623.

Lesieur, H. R. (2002). Epidemiological surveys if pathological gamblers: critique and sugestions for modification, en Marotta, J. J., Cornelius, J. A. y Eadington, W. R. (eds.). The downside: problem and pathological gambling, Reno (Ne): Institute for the study of the gambling and commercial gaming, 325-338.

Lesieur, H. R. y Blume, S. B. (1987). The South Oaks Gambling Screen (SOGS). A new instrument for the identification of pathological gamblers, American Journal of Psychiatry, 144, 1184-1188.

Lesieur, H. R. y Blume, S. B. (1993). Revising the South Oaks Gambling Screen in different setting, Journal of Gambling Studies, 9 (3), 213-223.

Lesiuer, H. R. y Rosenthal, R. J. (1998). Pathological gambling, a review of the literature, prepared for the American Psychiatric Association Task Force on DSM-IV Committee on disorders of impulse control not elsewhere classified, Journal of Gambling Studies, 7, 5-39.

Lloyd, J., Doll, H., Hawton, W. H., Geddes, J. R., Goodwin, G. M. y Rogers, R. D. (2010). How psychological symptoms delate to different motivations for gambling, An online study of Internet gamblers, Biological Psychiatry, 68 (8), 733-740, 2010b.

López Jiménez, A., Elboj Saso, C. y Lorente Tomás, A. (1999). Los juegos de azar. Juego social y ludopatía, Zaragoza: Universidad de Zaragoza y Diputación General de Aragón. Online: http://bases.cortesaragon.es/bases/NDocumen.nsf/0/b d00e58ea4c2715ec12576500032bbd0/\$FILE/ JUEG0+Y+LUDOPAT\%C3\%8DA.pdf

Mason, K. (2009). A focus on problem gambling: results of the 2006/07 New Zealand health survey, Wellington: Ministry of Health.
MarketQuest Research (2009). Newfoundland and Labrador Gambling Prevalence Study, St. Jonh's, (NL): Department of Health and Community Services.

McMillen, J., Marshall, D., Ahmed, E. y Wenzel, M. (2004). 2003 Victorian longitudinal community attitudes survey, The Centre for Gambling Research, Australia National University.

Neal, P., Delfabbro, P. y O'Neil, M. (2005). Problem gambling and harm, towards a national definition. Gambling Research Australia, Melbourne.

Ochoa, E. y Labrador, F. J. (1994). El juego patológico, Barcelona: Plaza y Janés.

Petry, N. y Weinstock, J. (2007). Internet gambling in common in college students and associated with poor mental health, American Journal on Addictions, 16, 325-330.

Potenza, M. N., Hollander, E. (2002). Pathologic gambling and impulse control disorders, en David, K. L., Charney, D., Coyle, J. T., Nemeroff, C. Neuropsychopharmacology: The Fifth Generation of Progress, American College of Neuropsychopharmacology.

Potenza, M. N., Xian, H. Shah, K., Scherrer, J. E. y Eisen, S. A. (2005). Shared genetic contributions to pathological gambling and major depression in men, Archives of General Psychiatry, 62, 1015-1021.

Ramírez, J. N., Cabanes, M., Carbonero, M., Castilla, R., Duarte, J. y Gutiérrez, B. (1999). Análisis del sector de las máquinas recreativas tipos " $A$ " $y$ " $B$ ". Estimación y análisis de la proporción de Iudópatas en Andalucía, Córdoba: Anmare.

Reilly, C., Smith, N. (2013). The evolving definition of pathological gambling in the DSM-5, National Center for Responsible Gaming.

Robins, L. N., Helzer, J. E., Croughan, J. L. y Ratcliff, K. S. (1981), National Institute of Mental Health Diagnostic Interview Schedule: its history, characteristics and validity, Arch. Gen. Psychiatry, 38 (4), 381-9.

Rönnberg, S., Volberg, R. A., Aabbott, M. W., Moore, W. L., Andre'n, A., Munck, I., Johnson, J., Nilsson, T. y Svensson, 0 (1999). Gambling and problem gambling in Sweden, Stockholm: Report to the National institute of Public Health on Gambling. Rosenthal, R. J. (1992). Pathological gambling, Psychiatric Annual, 22, 72-78. 
Ross, D. (2012). Addictive, impulsive and other counter-normative consumption, en Wells, V. y Foxall, G. Handbook of developments in consumer behaviour, Chetelhan UK-Northampton MaUSA, Edward Elgar.

Rotgers, F. y Christner, R. W. Addictive behaviorNonsubstance abuse, en Freeman, A., Felgoise, S. H., Nezu, A. M., Nezu, C. M, Reinecke, M. A. (2005). Encyclopedia of Cognitive Behavior Therapy, New York: Springer, 4-8.

Sáiz-Ruiz, J. e Ibáñez, A. (1999). Las bases neurobiológicas del juego patológico, Anales de Psicología, vol. 30, 4, 47-65.

Salinas, J. M. (2002). I/ Estudio sobre prevalencia de los jugadores de azar en Andalucía, Granada, Federación Andaluza de Jugadores de Azar Rehabilitados.

Salinas, J. M. (2004). Instrumentos de diagnóstico y screening del juego patológico, Salud y drogas, vol. 4, 2.

Sandven, U. H. (2007). The present situation in Norway, Presentation at the $6^{\text {th }}$. Nordic Conference on Gambling and Policy Issues, Copenhagen.

Santos Cansado, J. A. (coord.) (2012). Manual de intervención en juego patológico, Mérida: Servicio Extremeño de Salud.

Sendín, Federico (sd). Introducción al muestreo, apuntes y material de trabajo destinados al curso CIM de AEDEMO. Barcelona.

Shaffer, H. J. (2005). From disabling to enabling the public interest: National transitions from gambling exposure to adaptation and selfregulation, Addiction, 100, 1227-1229.

School of Urban Affairs and Public Policy (2002). The costs and consequences of gambling in the State of Delaware, Health and social services, Division of Substance Abuse and Mental Health.

Shapira, N. A., Ferguson, M. A., Frost-Pineda, K. y Gold, M. S. (2002). Gambling and problem gambling prevalence among adults in Florida, Report to the Florida Council on Compulsive Gambling.

Shaffer, H. J. y Hall, M. N. (2002). The natural history of gambling and drinking problems among casino employees, Journal of Social Psychology, 142 (4), 405-424.
Shafer, H. J., Hall, M. N. y Van der Bilt, J. (1997). Estimating the prevalence of disordered behaviour in the United States and Canada. A metaanalysis, Boston (MA): Harvard Medical School Division on Addictions, Boston, MA.

Shafer, H. J., Hall, M. N. y Van der Bilt, J. (1999). Estimating the prevalence of disordered gambling behaviour in the United States and Canada. A research synthesis, American Journal of Public Health, 89 (9), 1369-1376.

Slutske, W. S., Jackson, K. M. y Sher, K. J. (2003). The natural history of problem gambling from age 18 to 29, Journal of Abnormal Psychology, 112 (2), 263-274.

Sproston, K., Hing, N. y Palankay, C. (2012). Prevalence of gambling and problem gambling in New South Wales, NSW Office of liquor, gaming and racing. Ogilvy Illumination. Strategic communications research.

Stevens, M., Young, M. (2008). Gambling screens and problem gambling estimates: a parallel psychometric assessment of the South Oaks Gambling Screen and Canadian Problem Gambling Index, Gambling Research. Vol. 20, 1, 13-36.

Stinchfield, R. (2002). Reliability, validity and classification accuracy of the South Oak Gambling Screen (SOGS), Addictive Behaviors, 27, 1-19.

Stinchfild, R. y Winters, K. C. (1998). Adolescent gambling: a review of prevalence, risk factors and health implications, Annals of the American Academy of Political and Social Science, 556, 172-186.

Tejeiro, R. (1998). El juego de azar en Algeciras (España): prevalencia y características sociodemográficas, Revista Española de Drogodependencias, 23, 53-74.

Volberg, R. (2002). The epidemiology of pathological gambling, Psychiatric Annals, 32, 171-178.

Volberg, R. (1999). Research methods in the epidemiology of pathological gambling: Development on the field and directions for the future, Anuario de Psicología, 30 (4), 33-46.

Volberg, R. (1996). Prevalence studies of problem gambling in the United States, Journal of Gambling Studies, 12, 111-118.

Volberg, R. A., Abbott, M. W., Rönnberg, S. y Munck, I. M. E. (2001). Prevalence and risk of patho- 
logical gambling in Sweden, Acta Psychiatrica Scandinavica, 2001, 104, 250-256.

Volberg, R. A. y Steadman, H. J. (1988). Refining prevalence estimates of pathological gambling, The American Journal of Psychiatry, 154 (4), 502-505.

Wardle, H., Moody, A., Spence, S., Orford, J., Volberg, R., Jotangia, D., Griffiths, M., Hussey, D. y Dobbie, F. (2011). British Gambling Prevalence Survey 2010, National Centre for Social Research, London.

Wiebe, J., Single, E. y Falkkowski-Ham, A. (2003).

Exploring the evolution of problem gambling: a one year follow-up study, Report to the Responsible Gambling Council (Ontario).

Williams, R. J., Belanger, Y. D. y Arthur, J. N. (2011). Gambling in Alberta: History, current status and socioeconomic impacts, Final Report to the Alberta Gaming Research institute, Edmonton (Al).

Williams, R. J., Volberg, R. A., Rhis, M. G. y Stevens, S. (2012). The Population prevalence of problem gambling. Methodological influences, standardized rates, jurisdictional differences, and worldwide trends, Rapport prepared for the Ontario Problem Gambling Research Centre and The 0ntario Ministry of Health and Long Time Care.

Winters, K. C., Specker, S. y Stinchfield, R. (1997). Brief manual for use of Diagnostic Interview for Gambling Severity, University of Minnesota Medical School, Minneapolis, MN.

Wood, R. J. y Williams, R. J. (2009). Internet gambling: prevalence, patterns, problems and policy options, Report for the Ontario Problem Gambling Research Centre, Guelph, Ontario.

ANEXO 1.

FICHA TÉCNICA DE LAS ENCUESTAS.

\begin{tabular}{|c|c|c|c|c|}
\hline & \multicolumn{2}{|c|}{ España (población) } & \multicolumn{2}{|c|}{ España (online) } \\
\hline & 2015 & 2016 & 2015 & 2016 \\
\hline Universo & \multicolumn{2}{|c|}{ Residentes en España de 18 a 75 años } & \multicolumn{2}{|c|}{ Jugadores online en los dos últimos meses } \\
\hline Dimensión Muestra & 1002 & 1012 & 469 & 616 \\
\hline Margen de error: & $\pm 3,2 \%$ & $\pm 3,2 \%$ & $4,6 \%$ & $\pm 4,0 \%$ \\
\hline Cuestionario & \multicolumn{2}{|l|}{$10 / 15$ minutos } & \multicolumn{2}{|l|}{10 minutos } \\
\hline Tipo de entrevista & \multicolumn{2}{|l|}{ Telefónica } & \multicolumn{2}{|c|}{ Autoadministrada online } \\
\hline Muestreo & \multicolumn{2}{|c|}{$\begin{array}{l}\text { Proporcional a la población, por zonas } \\
\text { geográficas y hábitat. Cuotas de sexo, } \\
\text { edad y actividad }\end{array}$} & \multicolumn{2}{|c|}{$\begin{array}{l}\text { Cuotas de sexo y edad, según datos DGOJ } \\
\text { en } 2016 \text {. En } 2015 \text {, cuotas flexibles en } \\
\text { función de los datos sobre los jugadores } \\
\text { online obtenidos en el trabajo de campo } \\
\text { sobre la población entre } 18 \text { y } 75 \text { años. }\end{array}$} \\
\hline Fecha de realización & 13-20 / enero & 20-27 / enero & 6-12 / febrero & 19-27 / enero \\
\hline Red de campo & \multicolumn{2}{|l|}{ Millward Brown } & \multicolumn{2}{|l|}{ NetQuest } \\
\hline
\end{tabular}


ANEXO 2

\section{REFERENCIAS BIBLIOGRÁFICAS DE LA TABLA 13}

Ordenación por países y regiones

Alemania: (2015). Federal Center of Health Education (BZgA), (2016). Glücksspielverhalten und Glücksspielsucht in Deutschland. Ergebnisse des Surveys 2015 und Trends. Köln, Bundeszentrale für gesundheitliche Aufklärung.

Argentina (Área Metropolitana de Buenos Aires) (2015). Auguste, S. (2016). La ludopatía en Argentina, Buenos Aires: Universidad Torcuato Di Tella.

Australian Capital Territory (Au) (2009). Gambling and Racing Commission. The Australian National University, (2011). The ACT gambling prevalence study, Canberra.

New South Wales (Au). Sproston, K. y otros. Ver bibliografía.

Queensland (Au) (2012). Department of Justice and Attorney General (2012). Queensland Household gambling survey.

South Australia (Au) (2012). Department for Communities and Social inclusion (2013), Gambling prevalence in South Australia, Adelaida (SA).

Victoria (Au) (2012). Victoria Responsible Gambling Foundation (2012). The Victoria gambling study, Melbourne.

Canadá: Wood, R. T. y Williams, R. J. (2009). Ver bibliografía.

Corea del Sur (2011). Williams, R. J., Lee, C-K. y Back, K. J. (2011). Prevalence and nature of gambling and problem gambling in South Korea.

España: Referencias en la Tabla 1.

Estados Unidos (2008). Kessler, R., Hwang, I., La Brie, R., Petukhova, M., Sampson, H., Winters, K. (2008): DSM IV pathological gambling in the National comorbidity survey replication, Psychological Medicine, 38, 1351-1360.

Finlandia (2012). Raisamo, S. U., Mäkelä, P., SaIonen, A. H., Lintonen, T. P. (2014). The extend and distribution of gambling harm in Finland as assessed by Problem Gambling Severity Index, European Journal of Public Health, 25, 4, 716-722.
Turja, T., Halme, J., Mervola, M., Järvinen-Tassopoulos, J. y Ronkainen, J-E. (2012). Suomalaisten Rahapelaaminen 2011 (Finnish Gambling 2011), Helsinki: National Institute for Health and Welfare.

Francia (2014). Costes, J-M., Eroukmanoff, V., Richard, J-B., Tovar, M-L. (2015). Les jeux d'argent et de hasard en France en 2014, Observatoire des jeux, Inpes, París.

Gran Bretaña (2010). Wardle, H. y otros. Ver bibliografía.

Gales (2015). Gambling Commission (2015). Welsh Problem Gambling Survey 2015.

Irlanda del Norte (2010). Department for Social Development (NI) (2010). Northern Ireland gambling prevalence survey 2010, Belfast.

Hong Kong (2011). Hong Kong Polytechnic University. Department of Applied Social Sciences. (2012). A study of Hong Kong People's Participation in Gambling Activities, Commisioned by the Secretary for Home Affairs, Government of Hong Kong Special Administrative Region.

Hungría (2007). Kun, B., Bal'azs, H., Arnold, P., Paksi, B., Demetrovics, Z. (2011), Gambling in westerns and Eastern Europe: The example of Hungary, Journal of Gambling Studies. Doi:10.1007/s10889-011-9242-4.

Islandia (2007). Olason, D. T. (2009). Gambling and problem gambling studies among Nordic adults: Are they comparable? $7^{\text {th }}$ Nordic Conference, Helsinki, Finland.

Italia (2012). Ipsos-La Sapienza (Universitá de Roma) (2013). II fenómeno del gambling. I giocatori e l'evoluzione nel contesto italiano, Rapporto di ricerca 2012, Roma. Power Point.

Macao (2005): Ka-Chio Fong, D., Ozorio, B (2005). Gambling participation and prevalence estimates of pathological gambling in a Far East City: Macao. UNLV Gambling Research Review Journal, 9 (2), 15-28.

Noruega (2010). Pran, K. R., Ukkelberg, A. (2010), Spillevaner og spilleproblemer I befolkningen 2010, Synovate Norge.

Nueva Zelanda (2012). Abbot, M., Bellringer, M., Garret, N., Mundy-McPherson, S. (2014): New Zealand 2012 National Gambling Study: Gambling harm and problem Gambling, Wellinton, Ministry of Health. 
Singapur (2011). National Council of Problem Gambling (2012). Report of survey on participation in gambling activities among Singapore residents, 2011, Singapore.

Suecia (2009). Swedish National institute of Public Health (2011). Swedish longitudinal gambling study. Report no. 3.

Suiza (2007). Bondolfi, G. y otros (2008). Ver bibliografía.

Brodbeck, J., Durrenberger, S., Znoj, H (2007). Baseline study: Prevalences and consumption of games of change and their influence on the diagnosis of pathological gambling, University of Bern (Sw).

Sudáfrica (2008). Ross, D., Barr, G., Collins, P., Dellis, A., Hofmeyr, A., Kincaid, H., Rousseau, J., Schuhr, C., Visser, M., Vichinich, R. (2010). Summary of basic data from the National Urban Prevalence Study of Gambling Behaviour, The Research Division of the National Responsible Gambling Programme. 